Article

\title{
An Inducible System for Silencing Establishment Reveals a Stepwise Mechanism in Which Anchoring at the Nuclear Periphery Precedes Heterochromatin Formation
}

\author{
Isabelle Loïodice ${ }^{1}$, Mickael Garnier ${ }^{1}$, Ivaylo Nikolov ${ }^{1}$ and Angela Taddei ${ }^{1,2, *}$ \\ 1 Nuclear Dynamics Unit, CNRS, Institut Curie, PSL University, Sorbonne Université, 75005 Paris, France; \\ isabelle.loiodice@curie.fr (I.L.); mickael.garnier@curie.fr (M.G.); ivaylo.l.nikolov@gmail.com (I.N.) \\ 2 Cogitamus Laboratory, F-75005 Paris, France \\ * Correspondence: angela.taddei@curie.fr
}

Citation: Loïodice, I.; Garnier, M.; Nikolov, I.; Taddei, A. An Inducible System for Silencing Establishment Reveals a Stepwise Mechanism in Which Anchoring at the Nuclear Periphery Precedes Heterochromatin Formation. Cells 2021, 10, 2810. https://doi.org/10.3390/cells10112810

Academic Editor: Peter Askjaer

Received: 4 October 2021

Accepted: 13 October 2021

Published: 20 October 2021

Publisher's Note: MDPI stays neutral with regard to jurisdictional claims in published maps and institutional affiliations.

Copyright: (c) 2021 by the authors. Licensee MDPI, Basel, Switzerland. This article is an open access article distributed under the terms and conditions of the Creative Commons Attribution (CC BY) license (https:// creativecommons.org/licenses/by/ $4.0 /)$.

\begin{abstract}
In eukaryotic cells, silent chromatin is mainly found at the nuclear periphery forming subnuclear compartments that favor silencing establishment. Here, we set up an inducible system to monitor silencing establishment at an ectopic locus in relation with its subnuclear localization in budding yeast. We previously showed that introducing LacI bound lacO arrays in proximity to gene flanked by $H M L$ silencers favors the recruitment of the yeast silencing complex SIR at this locus, leading to its silencing and anchoring at the nuclear periphery. Using an inducible version of this system, we show that silencing establishment is a stepwise process occurring over several cell cycles, with the progressive recruitment of the SIR complex. In contrast, we observed a rapid, SIR-independent perinuclear anchoring, induced by the high amount of LacI binding at the lac $O$ array leading to nucleosome eviction at this array and to the phosphorylation of $\mathrm{H} 2 \mathrm{~A}$ in the neighboring nucleosomes by Mec1 kinase. While the initial phosphorylation of H2A (H2A-P) and perinuclear anchoring are independent of the SIR complex, its latter recruitment stabilizes H2A-P and reinforces the perinuclear anchoring. Finally, we showed that Sir3 spreading stabilizes nucleosomes and limits the access of specific DNA-binding protein to DNA.
\end{abstract}

Keywords: chromatin; heterochromatin; nuclear organization; gene regulation; gene silencing; FROS

\section{Introduction}

In eukaryotic cells, discrete regions of the genome assume heritable chromatin structures that silence genes located in these regions. In most organisms, and most cell types, silent chromatin or heterochromatin is enriched at the nuclear periphery forming subnuclear compartments where general repressors of transcription concentrate, thus favoring silencing establishment at the nuclear periphery [1,2].

In budding yeast, silent chromatin is mainly found in subtelomeric regions, at the two cryptic mating type loci (HML and HMR) and at the ribosomal DNA locus. In cycling cells, telomeres and $H M$ loci are preferentially located at the nuclear envelope, forming repressive domains that sequester the yeast silencing factors (SIRs) [3] in a manner comparable to heterochromatic chromocenters, which sequester HP1 in metazoans [1,4].

Similarly to HP1, the yeast silencing complex SIR2/3/4 is able to spread along the chromatin and to repress gene transcription owing to the histone deacetylase activity of Sir2 that creates a binding surface for Sir3 [3]. This spreading is limited by the amount of Sir3 and by the methylation of H3K79, which prevents Sir3 binding to nucleosomes [5].

At telomeres, silencing is nucleated at the subtelomeric TG1-3 repeats by the binding of the transcription factor Rap1 that contains binding sites for the silencing factors Sir3 and Sir4 at its C-terminal end. At the cryptic mating type loci, recruitment of the SIR complex requires the presence of cis acting elements called silencers that are formed by combinations of binding sites for DNA binding proteins including Rap1, Orc1, and Abf1, 
which like Rap1 have other functions in the cell. A common feature of these factors is their strong ability to displace nucleosomes [6].

At the rDNA, Sir2 is recruited by Net1 that associates with the RNA-polymerase I and Fob1 that binds the rDNA replication fork barrier as part of the RENT complex [7]. While Sir2, Sir3, and Sir4 are all required for silencing at HM loci and subtelomeres, Sir2 only is required for silencing at the rDNA, although Sir3 is found associated with the rDNA by ChIP and live microscopy [8,9].

In addition to these canonical sites, Sir3 is also found by ChIP at few sites located on chromosome arms [5,10-12]. In contrast to the situation at HM loci and subtelomeres, Sir3 does not spread from these recruitment sites owing to the presence of H3K79me3 nucleosomes on the flanking regions [5]. What triggers the recruitment of Sir3 at these sites is not known. Even at canonical silenced loci, it is not clear how the combination of binding sites for factors with independent roles in the cell can create a silencer able to nucleate gene silencing. It is generally admitted that the property of silencers emerges from the close juxtaposition of factors that interact directly or indirectly with one or more Sir proteins: a sufficiently high local concentration of Sir proteins to sustain silencing being achieved only when multiple binding sites are combined. However, there is no clear evidence for Abf1 interaction with any of the SIR proteins, suggesting that other mechanisms must be involved.

An important feature of heterochromatin in budding yeast is the limiting amount of the silencing factors Sir2 and Sir3, leading to an intranuclear competition between their target sites [13-15]. Another consequence of the limiting amount of SIR protein is that the efficiency of $H M$ silencers is sensitive to the distance to telomeres, which are located in compartments of the nucleoplasm enriched in silencing factors [16]. Accordingly, reporter genes flanked by functional silencers are weakly repressed by the SIR complex when integrated away from a telomere [17-21].

We previously showed that introducing tight protein-DNA complexes such as LacI bound $\mathrm{lacO}$ arrays in proximity to a reporter gene favor the recruitment of the SIR complex in this context [17], acting as a proto-silencer promoting gene silencing and change in subnuclear localization.

As mentioned above, silent chromatin is preferentially associated with the nuclear periphery due to different anchoring pathways, one of them involving Sir4 association with a network of docking sites on the inner nuclear membrane [16]. Reciprocally, the artificial tethering of a gene to the nuclear periphery favors SIR-mediated silencing [22] due to the high local concentration of SIR protein in this area of the nucleus [21]. Peripheral anchoring and SIR spreading thus reinforce each other once heterochromatin is established. However, their respective contribution for the establishment of silent chromatin is not known.

Heterochromatin establishment has been mainly studied using different strategies, most of them using conditional or inducible SIR3 alleles [23-32], thus affecting all silent loci and possibly changing the stoichiometry of the SIR complex. Here, we developed and characterized an inducible system to study the establishment of silencing at a single ectopic locus, while silencing is already established at canonical loci, without affecting the levels of silencing factors. We used this system to explore the relationship between histone modifications, perinuclear anchoring, and silencing factor recruitment upon silencing establishment.

\section{Materials and Methods}

\subsection{Media and Growth Conditions}

Yeast cells were grown either in rich medium (YP) supplemented with $2 \%$ glucose; $3 \%$ raffinose or $2 \%$ galactose ( $\mathrm{wt} / \mathrm{vol}$ ) or in synthetic medium (YNBAS MP Biomedicals, Solon, OH, USA) plates or liquid enriched for $2 \times$ dropout CSM-TRP (MP Biomedicals) and supplemented with $2 \%$ glucose $(w / v)$ (referred as CSM-TRP plates or medium). All the strains were grown at $30{ }^{\circ} \mathrm{C}$ with shaking at $250 \mathrm{rpm}$. For galactose induction, YP was supplemented with 0,008\% adenine- $\mathrm{HCl}$ (SIGMA A8751, Sigma-Aldrich, St. Louis, MO, 
USA). Typically, (for Figures 1-6 but Figures 6F and S6B) on day one, cells were thawed on YPD or CSM-TRP plates; on day two, cells were grown $6 \mathrm{~h}$ to $7 \mathrm{~h}$ in liquid YPAD and diluted at $0.01 \mathrm{OD}_{600} \mathrm{~nm}$ overnight in YPA $3 \%$ raffinose; on day three, cells were diluted at $0,2 \mathrm{OD}_{600} \mathrm{~nm}$ in YPA 3\% raffinose for $2 \mathrm{~h}$ then split in two. Galactose ( $2 \%$ final) was added to one half of the culture for galactose induction, the other half was kept in the same medium YPA 3\% raffinose as a control, and cells were put back to grow. At the end of day 3 , both cultures were diluted at $0.005 \mathrm{OD}_{600} \mathrm{~nm}$ and grew overnight to obtain the $20 \mathrm{~h}$ (or $1200 \mathrm{~min}$ ) time point.

For Figures 6F and S6B, freshly thawed strains on CSM-TRP plates were grown $6 \mathrm{~h}$ to $7 \mathrm{~h}$ in liquid $2 \times \mathrm{CSM}$-TRP medium, then diluted at $0.01 \mathrm{OD}_{600} \mathrm{~nm}$ overnight in $2 \times$ CSM-TRP medium. The next day, cells were diluted at $0.2 \mathrm{OD}_{600} \mathrm{~nm}$ in $2 \times \mathrm{CSM}$-TRP medium and imaged at $1 \mathrm{OD}_{600} \mathrm{~nm}$.

\subsection{Strains}

The strains used in this study are listed in Supplementary Table S1. They are all derivatives of W303 [33], except for yAT1798, yAT1909, and yAT2142 (S288C, [34]). Gene deletions, insertions of alternative promoters, and gene tagging were performed by PCR-based gene targeting on plasmids from $[35,36]$.

\subsection{Plasmids}

The plasmid encoding the GFP-LacI construct to be integrated at the ADE2 locus (pAT378) was constructed as described below: a part of the ADE2 ORF (nucleotide144-950) was amplified by PCR from pAT147 (pEADEI from [18]) using primers oAT884 and oAT885 that contain, respectively, in their $5^{\prime}$ extremity a SacI and SacII cutting sites. The PCR product was digested and cloned into pAT123 [17] digested by SacI and SacII yielding pAT376. A second PCR product spanning from nucleotide 1278 of $A D E 2$ to nucleotide 2635 of RGA1 was amplified from pAT147 using primers oAT995 and oAT996 that contain, respectively, in their $5^{\prime}$ extremity a SacI and NaeI cutting sites. This PCR product was digested and cloned into pAT376 digested by SacI and NaeI to obtain pAT377. Then, pAT377 was cut by HindIII and religated to obtain the final plasmid pAT378. Another version of this plasmid encoding a LacI protein resistant to galactose (LacI R197K) was obtained by directed mutagenesis on pAT378 using primers oAT1221 and oAT1222, yielding pAT391. pAT390, another version of the GFP-LacIR plasmid that is integrating at the LEU2 locus, was obtained by directed mutagenesis on pAT123 using primers oAT1168 and oAT1169. See sequences of the primers in Supplementary Materials Table S2.

\subsection{ChIP and Quantitative PCR Analysis}

A total of $20 \mathrm{OD}_{600} \mathrm{~nm}$ equivalent of cells was fixed in $20 \mathrm{~mL}$ with $0.9 \%$ formaldehyde for $15 \mathrm{~min}$ at $30^{\circ} \mathrm{C}$, quenched with $0.125 \mathrm{M}$ glycine for $5 \mathrm{~min}$, and washed once in cold $1 \times$ TBS pH 7.6. Pellets were suspended in $1 \mathrm{~mL} 1 \times$ TBS, centrifuged again, and frozen in liquid nitrogen after removal of the supernatant for $-80^{\circ} \mathrm{C}$ storage. All the following steps were done at $4{ }^{\circ} \mathrm{C}$ unless indicated. Pellets were resuspended in $500 \mu \mathrm{L}$ of lysis buffer (0.01\% SDS, 1.1\% Triton X-100, $1.2 \mathrm{mM}$ EDTA pH8, $16.7 \mathrm{mM}$ Tris $\mathrm{pH} 8,167 \mathrm{mM} \mathrm{NaCl}$, $0.5 \%$ BSA, $0.02 \mathrm{~g} / \mathrm{L}$ tRNA, and $2.5 \mu \mathrm{L}$ of protease inhibitor from Sigma-Aldrich P1860, Sigma-Aldrich, St. Louis, MO, USA) and mechanically lysed using a FastPrep instrument (MP Biomedicals) with $0.5 \mathrm{~mm}$ zirconium beads (Biospec Products, Bartlesville, OK, USA): intensity 6,3 cycles of $30 \mathrm{~s}$ with $3 \mathrm{~min}$ incubation on ice in between cycles. The chromatin was fragmented to a mean size of $500 \mathrm{bp}$ by either sonication using the Bioruptor XL (Diagenode, Liège, Belgium) for $14 \mathrm{~min}$ at high power with $30 \mathrm{~s}$ on/30 s off, or sonication in the Bioruptor Pico at high intensity for 3 cycles of $30 \mathrm{~s}$ on/30 s off. The extracts were cleared by centrifugation for $5 \mathrm{~min}$ at 16,000 g. Ten microliters was kept to be used as input. Cleared lysates were incubated overnight with the antibody used for chromatin immunoprecipitation: $1 \mu \mathrm{L}$ of polyclonal antibody anti-Sir3 (Agro-bio) (Ruault et al. 2011), $2 \mu \mathrm{L}$ of custom made polyclonal anti-Sir4 (Proteogenix, Schiltigheim, France), $3 \mu \mathrm{L}$ of 
polyclonal anti-H2A (Active motif, 39,235), $1 \mu \mathrm{L}$ of polyclonal anti-Histone H2A (phospho S129) (Abcam, ab15083, Cambridge, UK), and $4 \mu \mathrm{L}$ of polyclonal anti-acetyl-Histone $\mathrm{H} 4$ (Lys16) (Merck-Millipore, 2073125, Burlington, MA, USA). The next day, $50 \mu \mathrm{L}$ of magnetic beads protein A (NEB) was added to the extracts/antibody mixture and incubated for $4 \mathrm{~h}$ at $4{ }^{\circ} \mathrm{C}$ on a rotating wheel. Then, the magnetic beads with the immunoprecipitated material were washed sequentially once with lysis buffer, twice with RIPA buffer $(0.1 \%$ SDS, $10 \mathrm{mM}$ Tris $\mathrm{pH} 7.6,1 \mathrm{mM}$ EDTA pH8, 0.1\% sodium deoxycholate, and $1 \%$ Triton $\mathrm{X}-100)$, twice with RIPA buffer supplemented with $300 \mathrm{mM} \mathrm{NaCl}$, twice in $\mathrm{LiCl}$ buffer $(250 \mathrm{mM} \mathrm{LiCl}, 0.5 \%$ NP-40, $0.5 \%$ sodium deoxycholate), once with $1 \times$ TE $0.2 \%$ Triton X-100, and a final was in $1 \times$ TE. The beads were then resuspended in $100 \mu \mathrm{L}$ of elution buffer and placed in an incubator at $65{ }^{\circ} \mathrm{C}$ with gentle agitation to elute the immunoprecipitated material from the beads. In the meantime, the inputs were diluted $1 / 10$ with elution buffer. A reversal cross-linking was performed by heating the samples, inputs and IP, overnight at $65^{\circ} \mathrm{C}$. Proteins were digested with Proteinase $\mathrm{K}(0.4 \mathrm{mg} / \mathrm{mL})$ in the presence of glycogen, and the remaining DNA was purified on QIAquick PCR purification columns. Finally, samples were treated with $29 \mu \mathrm{g} / \mathrm{mL}$ RNase A for $30 \mathrm{~min}$ at $37^{\circ} \mathrm{C}$ and used for quantitative PCR.

ChIP quantification was performed by quantitative PCR (qPCR) either on the 7900HT Fast Real-Time PCR (Applied Biosystems, Waltham, MA, USA) or on the QuantStudio 5 Real-Time PCR System (Applied Biosystems, Waltham, MA, USA). Sequences of interest were amplified using the SYBR Green PCR Master Mix (Applied Biosystems, Waltham, MA, USA) and the primers listed in Supplementary Table S2 on a dilution of immunoprecipitated DNA at $1 / 40$ and $1 / 80$ for the input DNA. PCR reactions were conducted using the following program: an initial denaturation at $95{ }^{\circ} \mathrm{C}$ for $10 \mathrm{~min}$ followed by 40 cycles $\left(95^{\circ} \mathrm{C}\right.$ for $15 \mathrm{~s}$ and $60^{\circ} \mathrm{C}$ for $30 \mathrm{~s}$ ). Each real-time PCR reaction was performed in triplicate. The signal from a given region was normalized to the one from the OGG1 control locus in immunoprecipitated and input DNA samples. Plots represent the mean value obtained for at least three independent experiments; data are shown as mean \pm s.e.m.; see Supplementary Table S3 for the number of experiments.

\subsection{Quantitative Transcript Analysis}

Total RNA was isolated from yeast using the RNeasy kit (Qiagen, Hilden, Germany) followed by a DNase treatment (Qiagen, 79254, Hilden, Germany) to remove contaminating DNA. First strand cDNA was prepared from $0.5 \mu \mathrm{g}$ of RNA and SuperScript ${ }^{\mathrm{TM}}$ III FirstStrand Synthesis SuperMix for qRT-PCR (ThermoFisher, 11752050, Waltham, MA, USA). Quantitative PCR was performed on a 1/50 dilution of the reverse transcript mix with a couple of primers designed in ADE2 and a couple of primers designed in ACT1 (sequences provided in Supplementary Materials Table S2). Values were normalized by ACT1, then, to account for the change in expression induced by galactose induction independently of LacI induction, a ratio was made between strains with LacI expression (wt const. yAT2059 and wt ind. yAT2078) and a strain without LacI expression (wt No LacI yAT2001).

\subsection{Microscopy}

The set of images from any given figure panel was acquired using identical acquisition parameters. For all fluorescent images, the axial (z) step is $200 \mathrm{~nm}$, and images shown are a maximum intensity projection. Images were acquired using a wide-field microscopy system driven by MetaMorph software version 7.8 (Molecular Devices, Silicon Valley, CA, USA) based on an inverted microscope (Nikon TE2000, Nikon, Minato City, Tokyo, Japan) equipped with a $100 \times \sim / 1.4 \mathrm{NA}$ immersion objective, a C-mos camera (ORCA-flash C11440, Hamamatsu, Japan), and either a xenon arc lamp (Sutter Instrument Co. Lambda LS, Novato, CA, USA) or a Spectra X light engine lamp (Lumencor, Inc, Beaverton, OR, USA). 


\subsection{Image Processing and Analysis}

Deconvolution of images acquired with the wide-field microscopy system was made using the Meinel algorithm in MetaMorph (eight iterations; sigma =0.8; frequency 3; MDS Analytical Technologies, Sunnyvale, CA, USA).

All the microscopy image quantifications were done using either a single Fiji macro or MATLAB scripts with an additional expert supervision step to ensure that only relevant data are included in the statistical analysis.

\subsection{Spot Localization}

The spot localizations are extracted using a Fiji macro applying Laplacian of Gaussian filters on pre-processed images followed by an h-maxima transform. Due to the presence of highly variable signals present inside the yeast vacuoles, no reliable segmentation of the nuclear periphery has been achieved. Hence, a manual intervention from an expert was implemented in order to define two locations on the nuclear membrane: the closest point to the detected spot and on the opposite side of the nucleus in order to measure, respectively, the distance to the edge of the nucleus and its radius. The expert can also filter out any detection error and define the cell cycle.

\subsection{Intensity Quantifications}

Two different schemes are used to extract the spot intensities. The first, encapsulated inside the same Fiji macro for the spot localization, sums the intensities-minus the image background measured as its median intensity-of all the pixels comprised inside a threeby-three-by-three cube centered around the detection.

The other approach was preferred for data without the need to quantify the spot locations inside nuclei; thus, the signals inside vacuoles were not deemed problematic. It relies on $\mathrm{qFOCI}$, a MATLAB application currently being developed to extract diverse information from yeast nuclei and the spots they may contain. With this method, the spots are fully segmented in 3D through multiple Hessian of Gaussian filters followed by an MSER detector [37] and their intensities are the total integrated pixel values. Finally, the previous Fiji macro was adapted to add cycle information for the cells of the detected spots and to discard any false detection.

\subsection{Statistical Analysis}

In order to assess the presence of statistically significant differences between two given yeast strains or incubation time, be it among the localizations or intensities, Kruskal-Wallis tests by ranks were performed [38]. This non-parametric method tests for the null hypothesis stating that the different sets of data originate for the same distribution. In the case of multiple comparisons, the $p$-values are corrected using Tukey's range test [39]. All statistical analyses are performed with a significance level of 0.01 to reject the null hypothesis.

\section{Results}

\subsection{An Inducible and Reversible System to Establish Gene Silencing at an Ectopic Locus}

As mentioned above, $H M L$-mediated gene repression is dependent on the proximity to telomeres [20]. When an ADE2 gene flanked by the $H M L$ silencers is introduced at the LYS2 locus located $339 \mathrm{~kb}$ away from telomeres (hereafter named lys2::E-ADE2-I locus), yeast colonies are white, indicative of $A D E 2$ expression [18]. However, introducing a LacI bound lacO array in proximity to this construct leads to the formation of pink colonies, indicative of $A D E 2$ repression [17]. Here we modified this strain, placing the GFP-LacIR, a galactose-resistant variant, under the control of the inducible GALS promoter to be able to tightly control its expression (Figure 1A). Cells bearing a wild-type or mutated allele of $A D E 2$ at its endogenous location are shown for comparison. As expected, in non-inducible conditions (glucose-containing medium) our inducible strain formed white colonies, similarly to a strain bearing no LacI gene (Figure 1B). In contrast, in inducible conditions (galactose-containing medium; Figure 1B) the inducible strain formed colonies 
as pink as the strain expressing GFP-LacIR under the control of the constitutive HIS3 promoter. Consistent with our previous work [17], cells deleted for SIR1, SIR3, or SIR4 formed white colonies confirming that the SIR complex repressed the lys2::E-ADE2-I locus, when the neighboring lacO array is bound by LacI proteins (Figure S1).

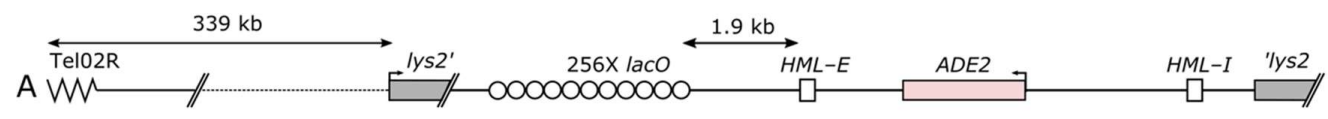

B

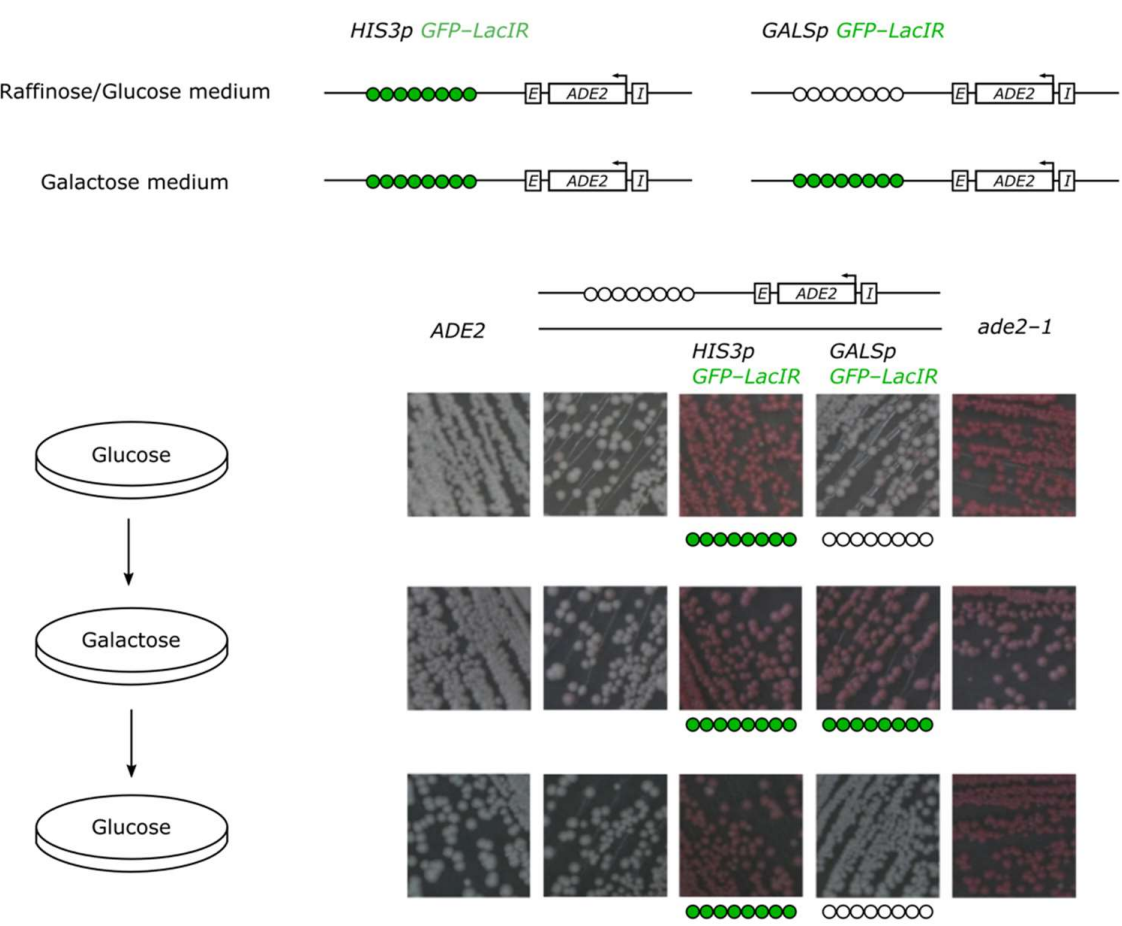

Figure 1. An inducible and reversible system to establish silencing at an ectopic locus. (A) Schematic representation of the construct used to study silencing establishment. A lacO array and the ADE2 reporter gene, flanked by $H M L$ silencers $E$ and $I$, were inserted at the LYS2 locus and referred to as the lys2::lacO E-ADE2-I locus. (B) Assessing the inducibility and reversibility of the system. The GFP-LacIR protein ( $\mathrm{R}$ for resistant to galactose) was expressed either under the constitutive HIS3 promoter (constitutive strains) or under the inducible GALS promoter (inducible strains). We followed the silencing of the $A D E 2$ gene by recording the color of the colonies streaked on plates (colonies are white when $A D E 2$ is expressed and pink when it is repressed). From left to right, pictures of a wild-type (WT) ADE2 strain as a control (yAT126), a strain bearing the lacO E-ADE2-I construct at LYS2 (yAT2001), a strain bearing the lacO E-ADE2-I construct at LYS2 and expressing the integrated GFP-LacIR under the constitutive HIS3 promoter (yAT2059) or under the inducible GALS promoter (yAT2078), and the WT ade2-1 strain unable to produce adenine as a control (yAT1). Strains were streaked on glucose (repressive condition for the GALS promoter), then from glucose to galactose (inducing conditions for the GALS promoter) and back to glucose plates.

When pink colonies from the inducing plate were streaked on non-inducible medium, cells formed white colonies, indicating that the silencing at the ectopic locus is not maintained in the absence of LacI expression (Figure 1B, bottom row).

Thus, LacI binding to $l a c O$ in vicinity to the $E-A D E 2-I$ reporter gene induces a reversible SIR-dependent repressed state.

\subsection{Perinuclear Anchoring Occurs independently of the Cell Cycle and Precedes Gene Silencing Establishment}

As mentioned above, LacI induced silencing of the lys2::lacO E-ADE2-I reporter is accompanied by its association with the nuclear periphery [17]. We used our inducible system to monitor the subnuclear localization of the locus and the expression of the ADE2 
reporter gene upon LacI induction. Cells were first grown in raffinose-containing medium before adding galactose to the medium. Faint GFP foci, corresponding to LacI binding the lacO array, were visible in $51 \%$ of cells 30 min after galactose induction in all phases of the cell cycle (determined using the transmission images based on the absence or presence of a bud), suggesting that LacI binding does not require a passage through $S$ phase. This percentage raised to $84 \%$ after $45 \mathrm{~min}$, and above $95 \%$ after $90 \mathrm{~min}$ of induction (Figure 2A). Foci intensity increased rapidly before saturating after three hours of induction in both G1 and S-G2 phase cells (Figures 2B and S2A). Intensity distribution of GFP spots in the strain expressing constitutively the LacI protein under the HIS3 promoter (here after the constitutive strain) is shown for comparison and is similar to the intensity observed after 45 min of induction (Kruskal-Wallis ANOVA test; $p=0.9$ ). We noticed that in the constitutive strain, intensities of the GFP spots were lower in the G1 phase than in the S-G2 phase (Figure S2A; $p=1 \times 10^{-10}$ ), as expected, since the lac $\mathrm{O}$ array replicates during the $S$ phase [40]. This difference was not observed after $30 \mathrm{~min}$ or $45 \mathrm{~min}$ of induction ( $p=0.38$ and 0.04 respectively), but it was statically significant after 90 min and even more obvious at later time points ( $p$ values ranging from $3 \times 10^{-6}$ to $10^{-10}$ ).

We monitored the relative distance of the lacO array to the nuclear periphery using Nup49-mCherry to label the nuclear envelope. At early time points of induction, the spatial distribution of these foci in the nuclear space was slightly biased toward the nuclear periphery (Figures 2C and S2B) without detectable changes between 30 and 45 min ( $p$-value $>0.99$ ). In contrast, $90 \mathrm{~min}$ after LacI induction, we observed a strong shift toward the nuclear envelope $\left(p=4 \times 10^{-6}\right)$. This distribution was similar after $180 \mathrm{~min}$ of induction $(>0.99)$ and even more peripheral after a long-term induction $\left(1200 \mathrm{~min}, p=3 \times 10^{-8}\right)$. At this stage the locus was more peripheral than in the strain expressing constitutively the LacI protein under the HIS3 promoter $\left(p=3 \times 10^{-3}\right)$. We observed a similar behavior when scoring the position of the locus in cells in the S-G2 phase, indicating that this perinuclear anchoring is mostly independent of the cell cycle (Figure S2C,D). In contrast, this perinuclear anchoring was not observed in cells without silencers at the LYS2 locus and expressing LacI under the weak constitutive HIS3 promoter, when grown in the same medium (galactose containing medium). We thus conclude that perinuclear anchoring of the LYS2 locus was not induced by growth in galactose medium but requires both the presence of silencer elements at this locus and LacI expression.

In parallel to the spatial localization of the locus, we monitored the establishment of the silencing at the $A D E 2$ reporter gene by measuring the ADE2 mRNA levels at different time points after LacI induction (Figure 2D). To account for the change in expression induced by galactose addition independently of LacI induction, we calculated the ratio of mRNA levels between the LacI inducible strain and a strain that did not carry a LacI gene for each time point. Before induction, ADE2 mRNA levels were 6.5-fold higher than in the strain with constant LacI expression. We observed a first $25 \%$ decrease in mRNA levels after 90 min of LacI induction, after which we observed little variations up to 360 min after induction. It was only after a long-term induction (1200 min) that mRNA levels reached the levels of the constitutive strain. Such a delay between LacI binding that reached the levels sufficient to induce silencing in the constitutive strain after $45 \mathrm{~min}$ of induction and the decrease in mRNA levels cannot be explained by the half-life of the ADE2 mRNA (which estimation varies between 13 and 40 min [41-44]).

Thus, complete repression required a long-term induction, although maximum LacI induction and binding were reached after $180 \mathrm{~min}$. In contrast, association with the nuclear periphery was to the level of the constitutively silent loci after $90 \mathrm{~min}$ of induction and further increased upon long-term induction. 
A HIS3p GFP-LaCIR

GALSp GFP-LaCIR
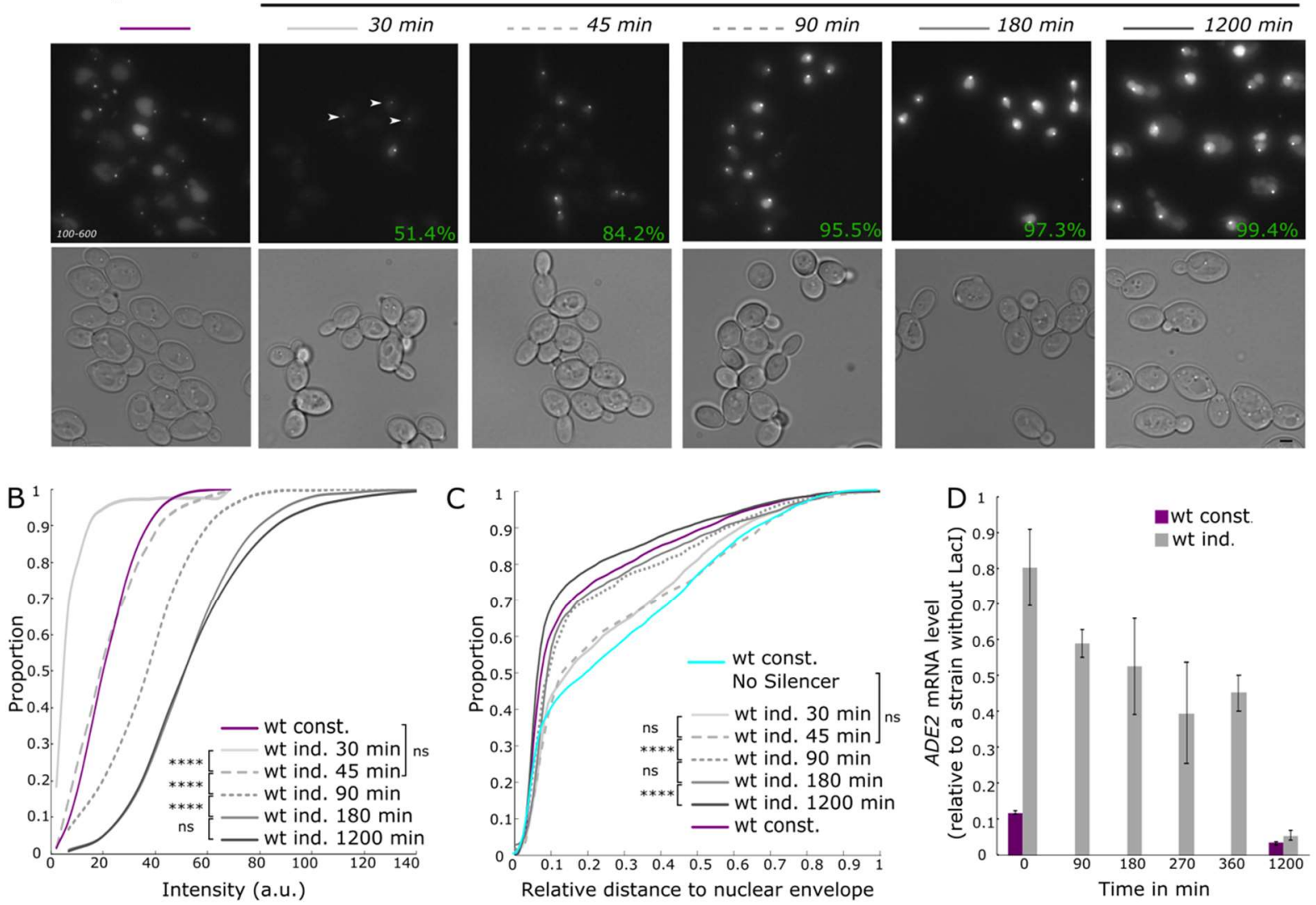

Figure 2. Perinuclear anchoring occurs independently of the Cell Cycle and precedes gene silencing establishment. (A) Representative fluorescent images (maximal projection of a Z-stack) and their corresponding transmission images of strains bearing the lys2::lacO E-ADE2-I locus and expressing GFP-LacIR either under the constitutive HIS3 promoter (wt const. yAT2059) or under the inducible GALS promoter (wt ind. yAT2078) at $30 \mathrm{~min}, 45 \mathrm{~min}, 90 \mathrm{~min}, 180 \mathrm{~min}$, and $1200 \mathrm{~min}$ after galactose induction. Percentage of cells with one spot is given for each time point after galactose induction of the GFP-LacIR protein. Bar, $2 \mu \mathrm{m}$. (B) Cumulative distributions of the intensity of GFP foci in G1 cells, in strains bearing the lys2::lacO E-ADE2-I locus and expressing GFP-LacIR either under the constitutive HIS3 promoter (wt const. yAT2059) or under the inducible GALS promoter (wt ind. yAT2078) at $30 \mathrm{~min}, 45 \mathrm{~min}, 90 \mathrm{~min}, 180 \mathrm{~min}$, and $1200 \mathrm{~min}$ after galactose induction of the GFP-LacIR protein. For statistics: $p>0.01$ is non-significant (ns), $p \leq 0.00001\left(^{(* * *)}\right.$ and see Table S4 for statistics. (C) Cumulative distributions of the relative distance of lys2::lacO E-ADE2-I or lys2::lacO locus to the nuclear periphery in G1 cells, in strains expressing GFP-LacIR either under the constitutive HIS3 promoter (wt const. yAT2059 and wt cont. No Silencer yAT3420) grown in galactose-containing medium or under the inducible GALS promoter (wt ind. yAT2078) at $30 \mathrm{~min}, 45 \mathrm{~min}, 90 \mathrm{~min}, 180 \mathrm{~min}$, and $1200 \mathrm{~min}$ after galactose induction of the GFP-LacIR protein. $p>0.01$ is non-significant (ns), $p \leq 0.00001\left(^{* * *}\right)$ and see Table S4 for statistics. (D) Graph showing the levels of $A D E 2$ mRNA (RT-qPCR) isolated from strains bearing lys2::lacO E-ADE2-I locus and expressing GFP-LacIR either under the constitutive HIS3 promoter (wt const. yAT2059) or under the inducible GALS promoter (wt ind. yAT2078) in raffinose $(t=0$, no induction) and after $90 \mathrm{~min}, 180 \mathrm{~min}, 270 \mathrm{~min}, 360 \mathrm{~min}$, and $1200 \mathrm{~min}$ after galactose induction. To account for changes in gene expression related to growth conditions independently of LacI induction, ADE2 mRNA levels were normalized to the $A D E 2$ mRNA levels of a strain not expressing the LacI protein (data are shown as mean \pm s.e.m.; see Table S3 for the number of experiments).

\subsection{Sir3 and Sir4 Accumulate over Several Cell Cycles following LacI Induction}

We next assessed the recruitment of Sir3 along the lys2::E-ADE2-I locus by chromatin immunoprecipitation (ChIP) analyzed by quantitative PCR, using primers located along the locus at different time points after LacI induction (Figure 3A). We previously showed a basal recruitment of Sir3 and Sir4 at the $E$ silencer and at the $A D E 2$ reporter gene flanked by the $H M L$ silencers, with or without an unbound lacO array located $1.9 \mathrm{~kb}$ upstream of 
the silencers (Dubarry et al., 2011). Consistently we observed a low level of Sir3 and Sir4 at these sites before LacI induction (Figures 3A and S3A). Upon induction, Sir3 and Sir4 binding gradually increased all along the locus.

A

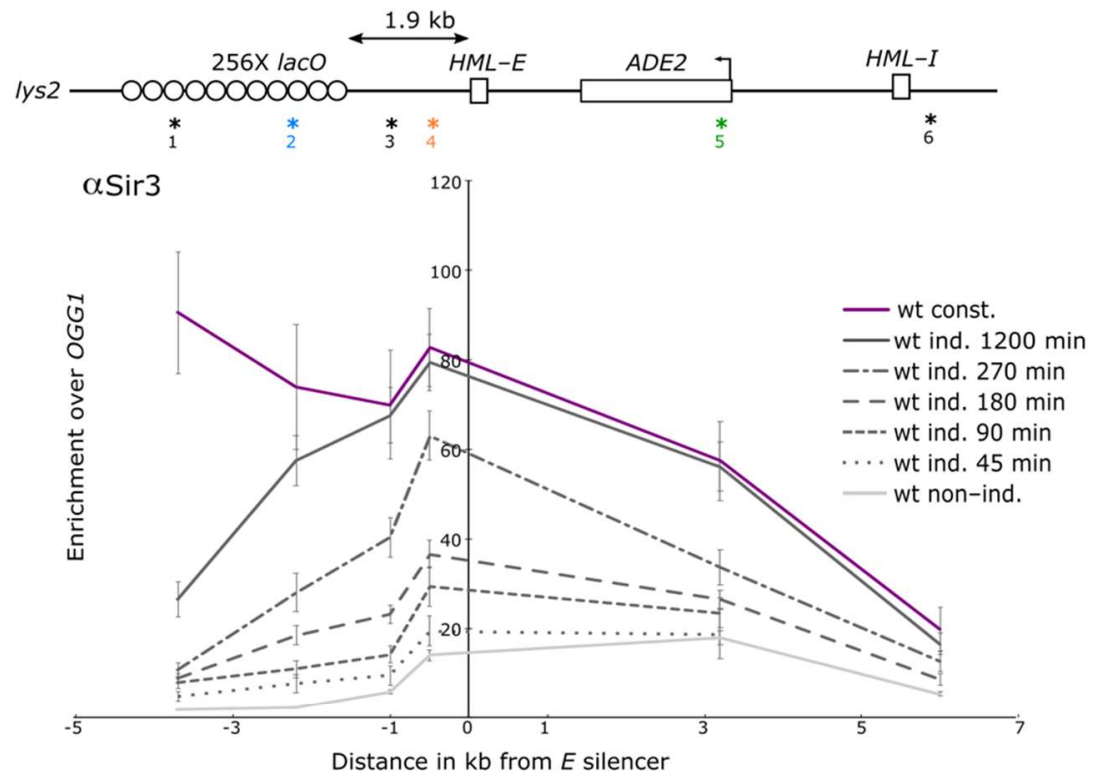

B

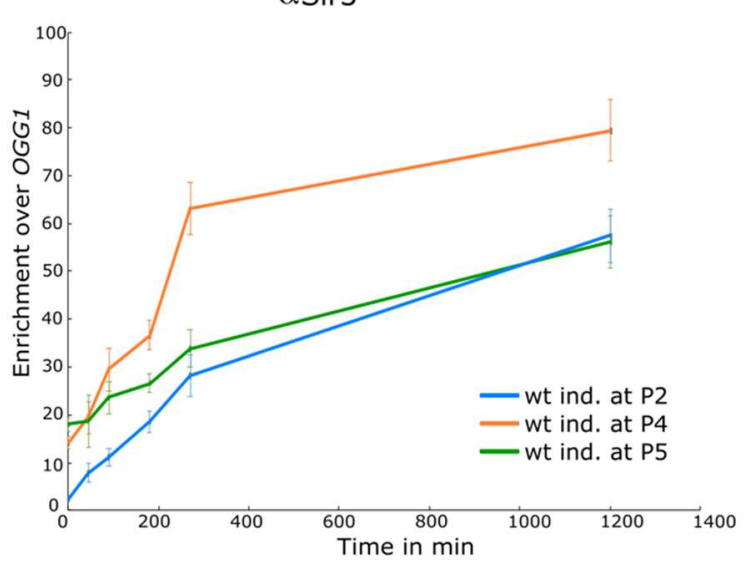

C

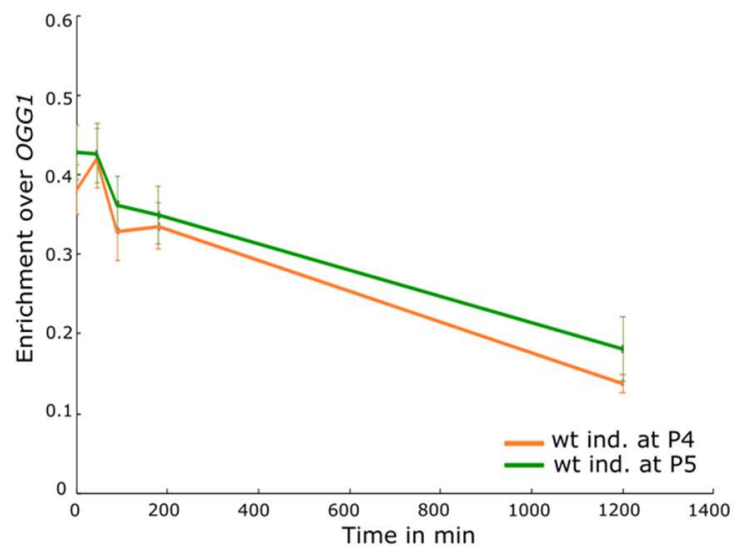

Figure 3. Heterochromatin formation takes place over several cell cycles. (A) Sir3 occupancy along the of lys2::lacO E-ADE2-I locus, probed by ChIP-qPCR using an anti-Sir3 antibody developed by us (Ruault et al., 2011), in strains expressing GFPLacIR either under the constitutive HIS3 promoter (wt const. yAT2059) or under the inducible GALS promoter (wt ind. yAT2078) in raffinose (no induction), or after $45 \mathrm{~min}, 90 \mathrm{~min}, 180 \mathrm{~min}, 270 \mathrm{~min}$, and $1200 \mathrm{~min}$ of galactose induction of the GFP-LacIR protein. The data were normalized over OGG1 (shown as mean \pm s.e.m.). (P1), (P2), (P3) (P4) amplicons are respectively located at $3.7 \mathrm{~kb}, 2.2 \mathrm{~kb}, 1 \mathrm{~kb}$, and $0.5 \mathrm{~kb}$ from the left side of the $E$ silencer, and (P5) and (P6) amplicons are located at $3.2 \mathrm{~kb}$ and $6 \mathrm{~kb}$ from the right side of the $E$ silencer. Amplicon positions are localized on the scheme of the locus with an asterisk and their respective number (see Table S3 for the number of experiments). (B) Sir3 enrichment over time at three particular sites of the lacO E-ADE2-I construct inserted at the LYS2 locus: at the lacO (P2), nearby the E silencer (P4), and in the body of ADE2 (P5) obtained by plotting data from Figure 3A. (C) H4K16 acetylation occupancy nearby the E silencer (P4) and in the body of ADE2 (P5) in a strain bearing the lys2::lacO E-ADE2-I locus, probed by ChIP-qPCR using an anti-acetyl-histone H4 (Lys16) antibody, in strains expressing GFP-LacIR under the inducible GALS promoter (wt ind. yAT2078) in raffinose (no induction), or after $45 \mathrm{~min}, 90 \mathrm{~min}, 180 \mathrm{~min}$, and $1200 \mathrm{~min}$ of galactose induction of the GFP-LacIR protein. The data were normalized over OGG1 (shown as mean \pm s.e.m.; see Table S3 for the number of experiments).

The fastest and highest recruitment was observed at the $E$ silencer (Figures $3 \mathrm{~A}, \mathrm{~B}$ and S3A), suggesting that Sir3 and Sir4 are first recruited at this site before spreading on the flanking 
regions. However, after 270 min of induction Sir3 enrichment was still below the one of the strains with constitutive LacI expression, this level being reached only after a long-term induction (Figure 3A,B). Probing Sir3 binding after $20 \mathrm{~h}, 48 \mathrm{~h}$, and $72 \mathrm{~h}$ of induction revealed that $\mathrm{Sir} 3$ recruitment did not show significant variation after $20 \mathrm{~h}$ (Figure S3B).

Focusing on the individual amplicons, we observed that Sir3 recruitment increased similarly at the $l a c O$ array and at the $E$ silencer during the first $3 \mathrm{~h}$ of induction and increased abruptly at the $E$ silencer after this time point, while Sir3 increased more progressively at the ADE2 gene over the time course (Figure 3B). These data suggest that the SIR complex is recruited both at the $\mathrm{lac} O$ array and the silencer at early time points before spreading on the $A D E 2$ gene.

The kinetics of Sir3 and Sir4 recruitment are in good agreement with the slow repression observed in Figure 2 and the kinetics of H4K16 deacetylation (Figures 3C and S3C,D). Indeed, H4K16 ChIP signal showed little variations during the first $180 \mathrm{~min}$ after LacI induction in the body of the reporter gene and reached the levels of the constitutive strain only after a long-term induction (Figures $3 \mathrm{C}$ and S3C,D). In contrast, we noticed an abrupt decrease in $\mathrm{H} 4 \mathrm{~K} 16$ acetylation along the lacO array, during the first 90 min following LacI induction (Figure S3C), even when normalizing the H4K16Ac ChIP signal by nucleosome occupancy (Figure S3D).

Altogether, our data show that heterochromatin formation and silencing establishment are slow processes taking place over several cell cycles. This is in contrast with the kinetic of perinuclear anchoring that occurs mainly during the first $90 \mathrm{~min}$ following LacI induction.

\subsection{Perinuclear Anchoring Is Partly Sir3 Independent}

The difference in kinetic of perinuclear anchoring and silencing factor recruitment suggests that perinuclear anchoring of the lys2::E-ADE2-I locus could occur independently of heterochromatin formation upon Lacl binding. To test this hypothesis, we monitored the distance of this locus to the nuclear periphery in SIR3 or SIR4 deleted strains (Figures Figure $4 \mathrm{~A}$ and $\mathrm{S} 4 \mathrm{~A}$ ). In the sir3 $\Delta$ strain, the localization was similar to the wt strain, when monitored 30-45 min after LacI induction $(p=0.96)$. After $3 \mathrm{~h}$ of induction the locus was shifted toward the nuclear periphery to a similar extent than in the wt strain $(p=0.9)$, but no further changes in localization were observed after a long-term induction in both $\operatorname{sir} 3 \Delta$ and $\operatorname{sir} 4 \Delta$ strains. Similar results were obtained in G1 or S-G2 cells (Figure S4B).

We thus observed an SIR-independent anchoring of the locus to the nuclear periphery following LacI induction. We next tested whether this perinuclear anchoring was also independent of the presence of a neighboring silencer element.

In the absence of silencers, the LYS2 locus also showed a more peripheral localization upon high LacI expression compared with a strain expressing the LacI protein under the weaker constitutive HIS3 promoter grown in the same medium (Figure 4B, $p=3.8 \times 10^{-9}$ ). The only difference between these two strains is the amount of LacI expression and binding to the $l a c O$ array, the median intensity of the lacO spot being 3.7-fold higher in the induced strain than in the constitutive strain (Figure 4C,D). Therefore, a high level of LacI binding is sufficient to induce a shift of the locus to the nuclear periphery independently of Sir3, Sir4, or the presence of silencer elements. However, this LacI induced Sir3-independent perinuclear anchoring is weaker than the one observed when the locus is silenced $\left(p=5 \times 10^{-4}\right)$. We thus conclude that the perinuclear anchoring of the lys2::lacO E-ADE2-I locus, following $\mathrm{LacI}$ binding to the lacO array, is partially independent of heterochromatin establishment. 
A

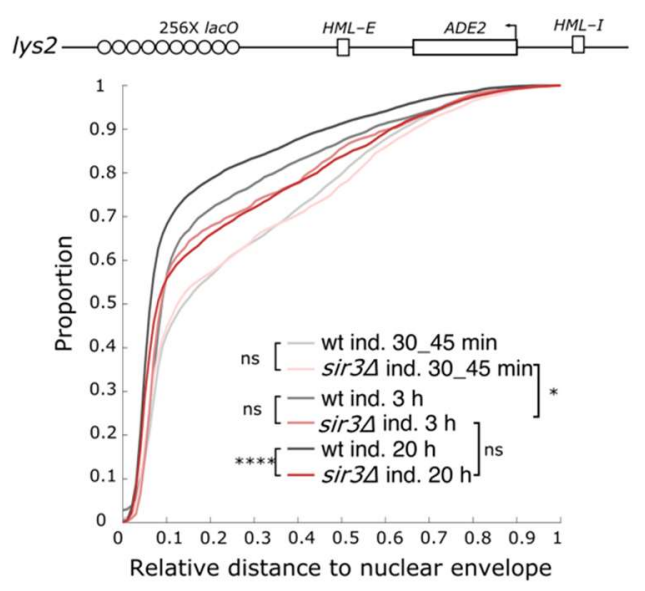

C

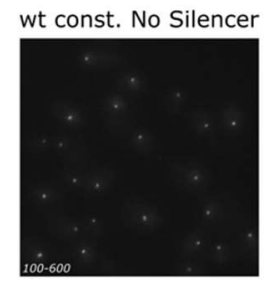

wt ind. $20 \mathrm{~h}$ No Silence

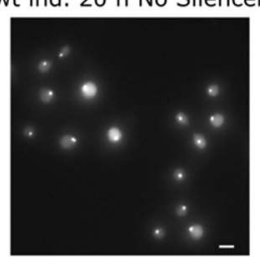

B

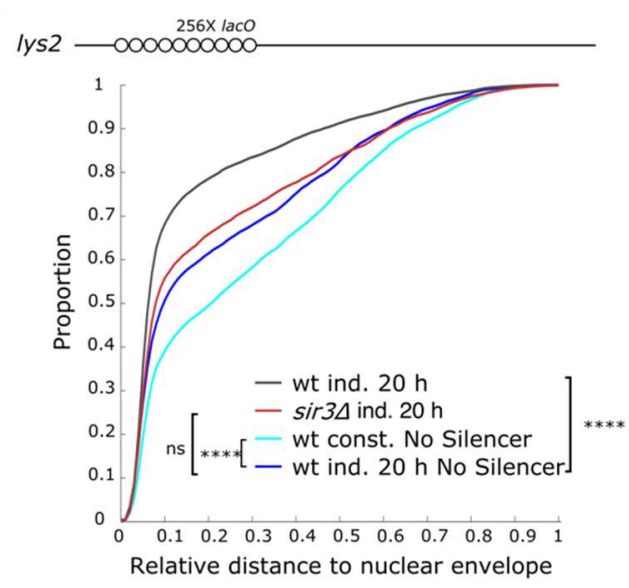

$\mathrm{D}$

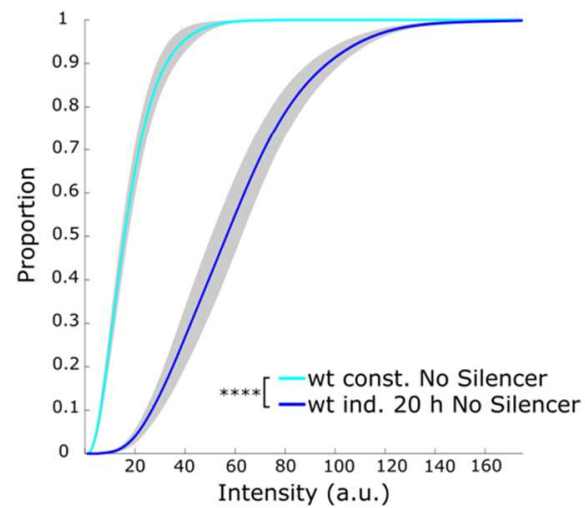

Figure 4. Perinuclear anchoring is partly Sir3 independent. (A) Cumulative distributions of the relative distance of the lys2::lacO E-ADE2-I locus to the nuclear periphery, in G1 cells, in strains expressing GFP-LacIR under the control of the GALS promoter in the WT (wt ind., yAT2078), and in a sir $3 \Delta$ strain (sir $3 \Delta$ ind., yAT2370) after $30-45 \mathrm{~min}, 3 \mathrm{~h}$, and $20 \mathrm{~h}$ of galactose induction. $p>0.01$ is non-significant (ns), $p \leq 0.01\left(^{*}\right), p \leq 0.00001\left(^{* * * *}\right)$ and see Table S4 for statistics. (B) Cumulative distributions of the relative distance of lys2::lacO E-ADE2-I locus to the nuclear periphery in G1 cells, in strains expressing GFP-LacIR under the inducible GALS promoter in the WT (wt ind. yAT2078), and in a sir3 $($ sir3 $\triangle$ ind., yAT2370) strains after $20 \mathrm{~h}$ of galactose induction. Cumulative distributions of the relative distance of the lys2::lacO array locus (No Silencer) to the nuclear periphery in G1 cell, in strains expressing GFP-LacIR either under the inducible GALS promoter (wt ind. No Silencer, yAT3471) after $20 \mathrm{~h}$ of galactose induction or under the constitutive HIS3 promoter grown in galactose (wt const. No Silencer, yAT3420). $p>0.01$ is non-significant (ns), $p \leq 0.00001{ }^{* * * *}$ ) and see Table S4 for statistics. (C) Representative fluorescent images (maximal projection of a Z-stack) of strains bearing the lys2::lacO array locus (No Silencer) and expressing GFP-LacIR either under the constitutive HIS3 promoter (wt const. No Silencer yAT3420) or under the inducible GALS promoter (wt ind. No Silencer yAT3471) at $20 \mathrm{~h}$ after galactose induction. Bar, $2 \mu \mathrm{m}$. (D) Cumulative distributions of the intensity of GFP foci in G1 cells, in strains bearing a lys2::lacO array locus (No Silencer) and expressing GFP-LacIR either under the constitutive HIS3 promoter (wt const. No Silencer yAT3420) or under the inducible GALS promoter (wt ind. No Silencer yAT3471) at $20 \mathrm{~h}$ after galactose induction. $p>0.01$ is non-significant (ns), $p \leq 0.01 p \leq 0.00001\left(^{* * * *}\right.$ ) and see Table S4 for statistics.

\subsection{LacI Binding Induces a Mec1 Dependent H2A-P}

We hypothesized that the Sir3-independent change in localization induced upon LacI binding could result from chromatin stress induced by the LacI binding to the lacO array. We thus set out to monitor the presence of histone H2A phosphorylation, a mark associated both with silent chromatin and DNA damage [45,46], at the lys2::lacO E-ADE2-I locus following LacI induction. To account for potential variation in H2A occupancy, we normalized the H2A phosphorylation (H2A-P) ChIP signal against total H2A ChIP performed on the same samples. 
The H2A-P/H2A signal increased with time after induction over the locus, with a faster and higher increase at the $E$ silencer, suggesting that $\mathrm{H} 2 \mathrm{~A}$ was first phosphorylated there before the mark spread on the flanking regions (Figures $5 \mathrm{~A}$ and $\mathrm{S} 5 \mathrm{~A}, \mathrm{~B}$ ).

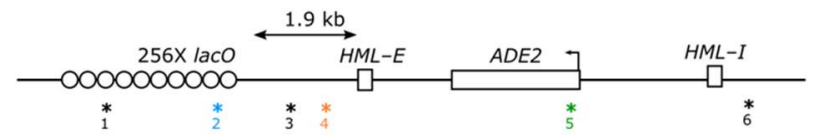

A

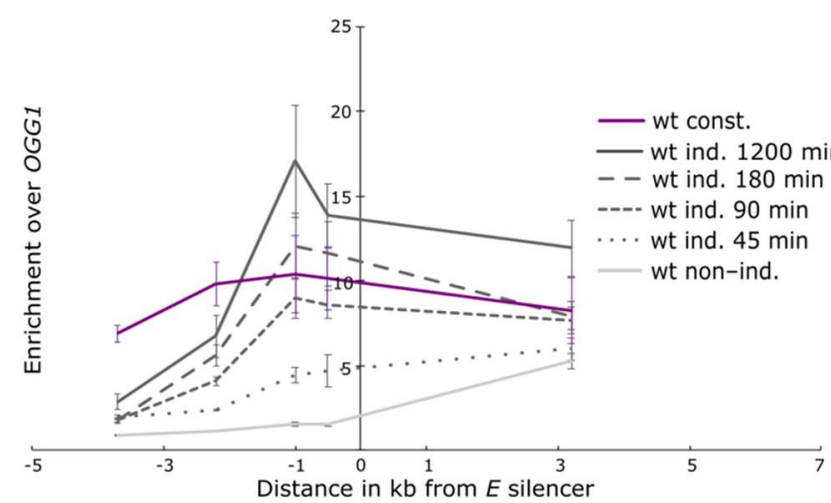

B

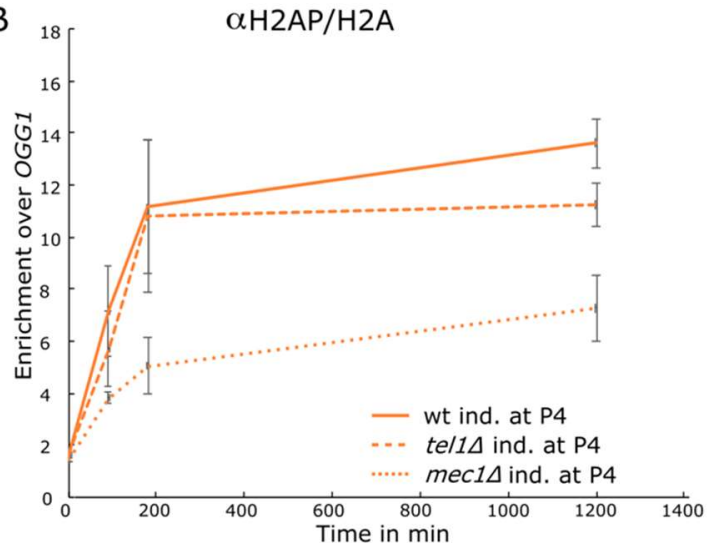

C

E

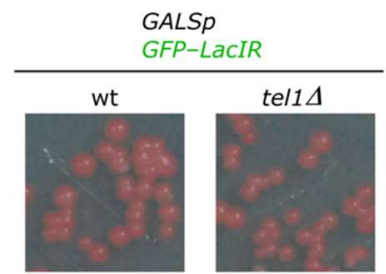

$\operatorname{mec} 1 \Delta$

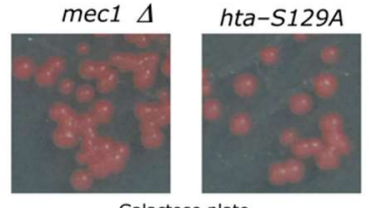

Galactose plate
D
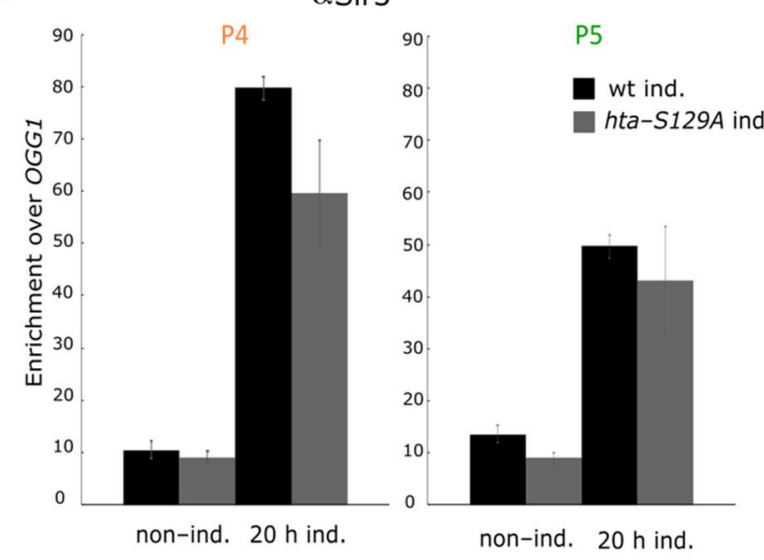

$\mathrm{F}$

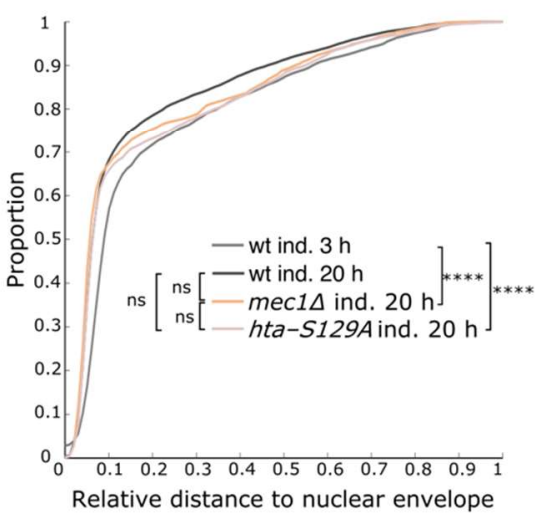

Figure 5. Mec1-dependent H2A phosphorylation induced by LacI binding and stabilized by Sir3 spreading. (A) H2A-S129 phosphorylation occupancy along the lys2::lacO E-ADE2-I locus probed by ChIP-qPCR using an anti-H2A Phospho-S129 antibody in strains expressing GFP-LacIR either under the constitutive HIS3 promoter (wt const. yAT2059) or under the inducible GALS promoter (wt ind. yAT2078) in raffinose (no induction), or after $45 \mathrm{~min}, 90 \mathrm{~min}, 180 \mathrm{~min}$, and $1200 \mathrm{~min}$ of galactose induction. The data were normalized over OGG1 and histone H2A ChIP signal (shown as mean \pm s.e.m.; see 
Table S3 for the number of experiments). (P1), (P2), (P3) (P4) amplicons are respectively located at $3.7 \mathrm{~kb}, 2.2 \mathrm{~kb}, 1 \mathrm{~kb}$, and $0.5 \mathrm{~kb}$ from the left side of the $E$ silencer, and (P5) and (P6) amplicons are located at $3.2 \mathrm{~kb}$ and $6 \mathrm{~kb}$ from the right side of the $E$ silencer, amplicon positions are localized with an asterisk and their respective number on the scheme of the locus. (B) H2A-S129 phosphorylation enrichment over time nearby the $E$ silencer (P4) in strains bearing the lys2::lacO E-ADE2-I locus, probed by ChIP-qPCR using an anti-H2A Phospho-S129 antibody, in strains expressing GFP-LacIR under GALS promoter in the WT (wt ind., yAT2078), in a tel1 $\Delta$ (tel1 $\Delta$ ind,. yAT2314), and in a mec1 $\Delta$ (mec1 $\Delta$ ind., yAT2904) strains in raffinose (no induction), or after $90 \mathrm{~min}, 180 \mathrm{~min}$, and $1200 \mathrm{~min}$ of galactose induction. The data were normalized over OGG1 and histone H2A ChIP signal (shown as mean \pm s.e.m.; see Table S3 for the number of experiments). (C) H2A-S129 phosphorylation enrichment over time nearby the $E$ silencer $(\mathrm{P} 4)$ in strains bearing the lys2::lacO E-ADE2-I locus, probed by ChIP-qPCR using an anti-H2A Phospho-S129 antibody, in strains expressing GFP-LacIR under GALS promoter in the WT (wt ind., yAT2078), and in a sir3 $\Delta$ (sir3 $\Delta$ ind,. yAT2370) strain in raffinose (no induction), or after $45 \mathrm{~min}, 90 \mathrm{~min}, 180 \mathrm{~min}$, and $1200 \mathrm{~min}$ of galactose induction. The data were normalized over OGG1 and histone H2A ChIP signal (shown as mean \pm s.e.m.; see Table S3 for the number of experiments). (D) Sir3 enrichment probed by ChIP-qPCR using Sir3 antibody nearby the $E$ silencer (P4) and at ADE2 (P5) in strains bearing the lys2::lacO E-ADE2-I locus, and expressing GFP-LacIR under the GALS promoter in the WT (wt ind., yAT2078), and in a hta-S129A (hta-S129A ind, yAT2965) strains in raffinose (no induction), and $20 \mathrm{~h}$ of galactose induction. The data were normalized over OGG1 (shown as mean \pm s.e.m.; see Table S3 for the number of experiments). (E) Pictures of yeast colonies bearing the lys2::lacO E-ADE2-I locus and expressing the GFP-LacIR under

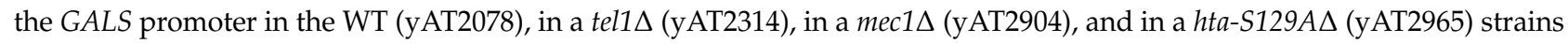
streaked on galactose plate. (F) Cumulative distributions of the relative distance of lys2::lacO E-ADE2-I locus to the nuclear periphery in G1 cells, in strains expressing GFP-LacIR under the GALS promoter in the WT (wt ind., yAT2078) strain after

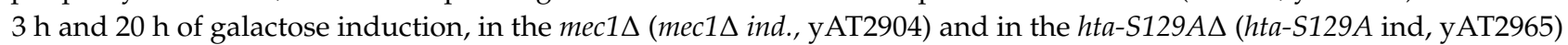
strains after $20 \mathrm{~h}$ of galactose induction. $p>0.01$ is non-significant (ns), $p \leq 0.00001\left({ }^{* * *}\right)$ and see Table S4 for statistics.

We next tested the respective contribution of the two H2A kinases Mec1 and Tel1 in this phosphorylation event since Tel1 is the main kinase responsible for H2A-P at telomeres ([45] and Figure S5C), whereas Mec1 is the main kinase acting at processed DSB [47]. While deleting TEL1 had no significant effect on the kinetic and level of H2A-P, deleting MEC1 led to a reduction in H2A-P (Figure 5B) upon LacI induction compared to the wild-type strain. However, we still observed a slow accumulation of H2A-P at the $E$ silencer in the absence of Mec1. This suggests that although Mec1 is the main kinase responsible for H2A-P at the E silencer upon LacI induction, Tel1 can at least partially substitute Mec1 activity.

\subsection{H2A-P Induced by LacI Binding Is Sir3 Independent but Is Stabilized by Sir3 Spreading}

As mentioned above, genome-wide studies showed a strong correlation between H2A-P and silent chromatin $[5,45,46]$.

The significant increase in $\mathrm{H} 2 \mathrm{~A}-\mathrm{P}$ at the $E$ silencer as soon as $45 \mathrm{~min}$ after induction, before any significant change in localization or in SIR recruitment occurred, indicated that this phosphorylation event could be SIR independent. We tested this hypothesis by monitoring H2A-P/H2A in a SIR3 deleted strain upon LacI induction. We observed a transient phosphorylation of $\mathrm{H} 2 \mathrm{~A}$ at the $E$ silencer and the lacO array in this strain (Figures 5C and S5E). This Sir3 independent, LacI induced H2A-P peaked 90 min after induction before slowly decreasing to reach basal levels upon a long-term induction at the $E$ silencer and a two-fold enrichment at the lacO array (Figures 5C and S5E). Thus, while the initiation of H2A-P is Sir3 independent, its accumulation depends on the presence of Sir3. These data suggest that H2A phosphorylation is an early event induced by LacI binding at the lacO array, and that this mark is later stabilized by Sir3 binding to nucleosomes. In good agreement with this hypothesis, we also observed a phosphorylation of $\mathrm{H} 2 \mathrm{~A}$ after $90 \mathrm{~min}$ of LacI induction at the same distance from the lacO array in the strain without silencer (Figure S5F).

$\mathrm{H} 2 \mathrm{~A}$ phosphorylation being the first event that we could detect at the silencer, we wondered whether this modification could be responsible for the recruitment of the SIR complex. We thus assayed the H2A-S129A ( $h t a-S 129 A$ ) mutant that could not be phosphorylated by the PI3 kinases Mec1 and Tel1 (Downs et al. 2000), for Sir3 recruitment. Although we observed a slight defect of Sir3 recruitment at the $E$ silencer after a long-term LacI induction, this was not the case at the $A D E 2$ gene (Figure 5D). Consistently, we did not 
observe any defect in silencing establishment upon LacI induction, neither in this strain nor in the MEC1 or TEL1 deleted strain (Figure 5E). Similarly, Sir3 recruitment was reduced in the H2A-S129A mutant strain $0.2 \mathrm{~kb}$ from the telomere $6 \mathrm{R}$ but unaffected in further distant subtelomeric regions (Figure S5G). Therefore, H2A-P may have a small contribution at the nucleation step but did not impact Sir3 spreading and the resulting gene silencing.

We next tested the impact of this modification on the perinuclear anchoring of the locus. Distributions of the locus in mec1 $1 \Delta$ or H2A-S129A expressing strains were similar to the distribution in the wild-type strain (Figure 5F, $p=0.76$ ). In the absence of Sir3, the localization of the locus was not significantly different in the absence or presence of this mutation, although we noticed a higher variability of the data in the double mutant $(p=0.8$; Figure $\mathrm{S} 5 \mathrm{H})$. Thus, $\mathrm{H} 2 \mathrm{~A}$ phosphorylation is not required for the perinuclear anchoring of the lys2::lacO E-ADE2-I locus upon LacI binding.

In summary, the H2A phosphorylation event observed 45 min after LacI binding on the lacO array is independent on Sir3 recruitment, and reciprocally, this modification is not required for Sir3 recruitment or the perinuclear anchoring of the locus. However, Sir3 recruitment that occurs later after LacI induction (starting at $90 \mathrm{~min}$ ) allows the stabilization of this mark, which then accumulates over the long-term induction.

\subsection{Sir3 Spreading Counteracts LacI-Induced Nucleosome Eviction and Limits LacI Binding}

Plotting H2A occupancy along the locus at different times after induction revealed a progressive decrease in H2A ChIP signal over the lac O array, from $45 \mathrm{~min}$ to $3 \mathrm{~h}$ after induction. At this time, H2A enrichment (over our reference locus, OGG1) was 3-fold lower than before LacI induction. However, we observed a slight but reproducible increase in $\mathrm{H} 2 \mathrm{~A}$ occupancy after long-term induction (Figure 6A,B). We wondered whether this late re-association of $\mathrm{H} 2 \mathrm{~A}$ could be related to the SIR spreading observed after long-term induction (Figure 3). Indeed, in the absence of Sir3, H2A eviction was stronger than in the wild-type strain $3 \mathrm{~h}$ after induction and remained stable after long-term LacI induction (Figure 6B). In the constitutive strain expressing a lower level of LacI, we observed a 20 to $25 \%$ decrease in $\mathrm{H} 2 \mathrm{~A}$ occupancy as compared with the signal obtained in the absence of LacI binding (Figure 6C). Again, in the absence of Sir3, the eviction was stronger, with H2A occupancy at the $l a c O$ array corresponding to $30 \%$ of the occupancy of the unbound array. A similar level of H2A ChIP signal was observed in a wt strain expressing the LacI under the HIS3 promoter but without silencer flanking the lacO array (Figure S6A). These results suggest that Sir3 spreading from the silencer stabilizes nucleosomes that are removed by LacI binding in the absence of Sir3.

It is noteworthy that canonical sites of SIR recruitment are also associated with nucleosome-poor regions such as the TG repeats, subtelomeric sequences, and the HM silencers. Indeed, H2A signal is rather low immediately adjacent to telomere 6R compared to other genomic sites ( $60 \%$ of the reference signal), and it is even lower in the absence of Sir3 (Figure 6D), indicating that Sir3 spreading stabilizes the nucleosome, in good agreement with previous work [48-50].

We then asked whether Sir3 spreading could prevent LacI binding at the lacO array. We thus compared the intensity of the GFP-LacI spot neighboring the E-ADE2-I reporter in wild-type or sir $3 \Delta$ strain expressing the LacI protein under the constitutive HIS3 promoter (Figure 6E). We observed that the intensity of the spots in G1 cells was higher in the absence of Sir3 $\left(p=10^{-10}\right.$, Figure 6 F). Similarly, GFP-LacI spots were brighter in the absence than in the presence of a neighboring silencer element (Figure S6B). We thus conclude that Sir3 spreading impairs LacI binding.

All together these data indicate that Sir3 stabilizes nucleosome and prevents the access of DNA binding factor to DNA. 


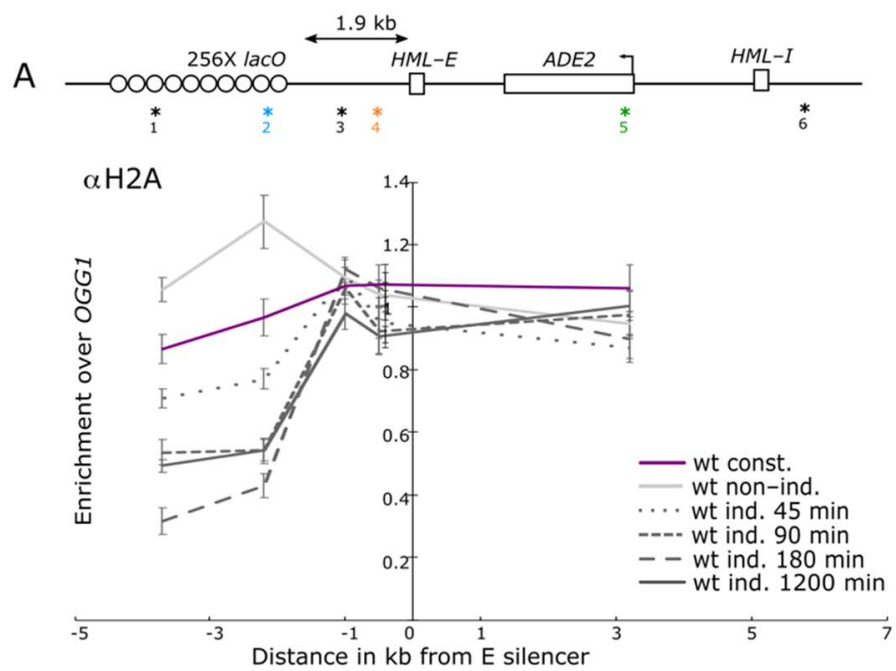

C $\alpha \mathrm{H} 2 \mathrm{~A}$

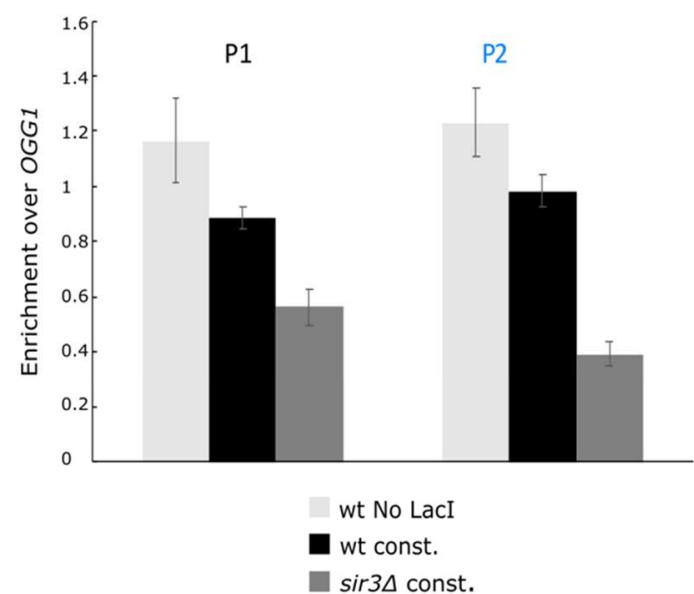

$\mathrm{E}$
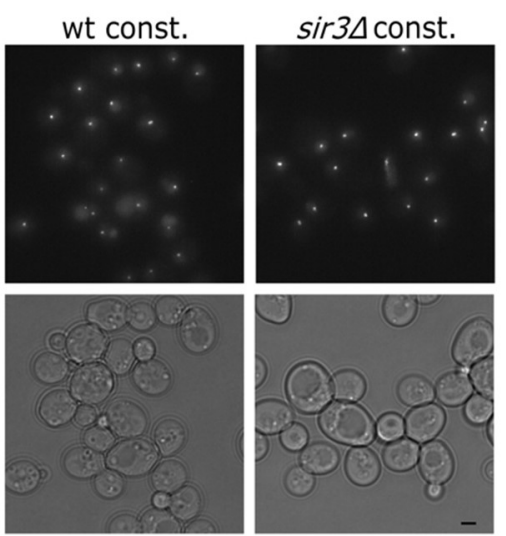

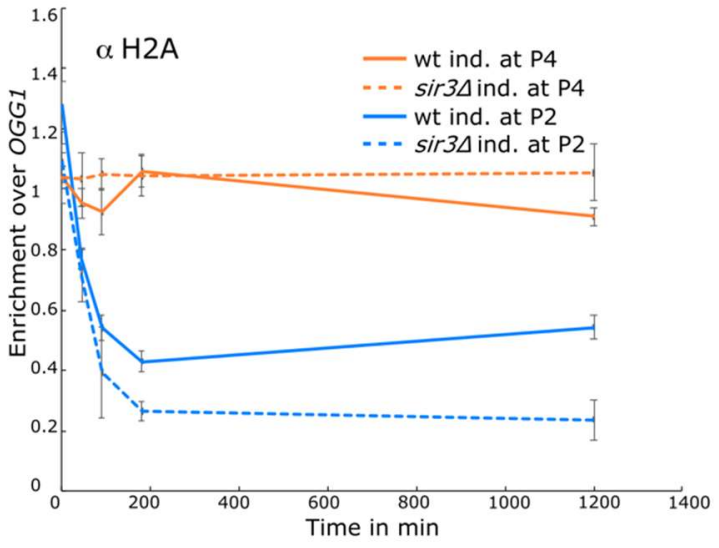

D $\quad \alpha \mathrm{H} 2 \mathrm{~A}$

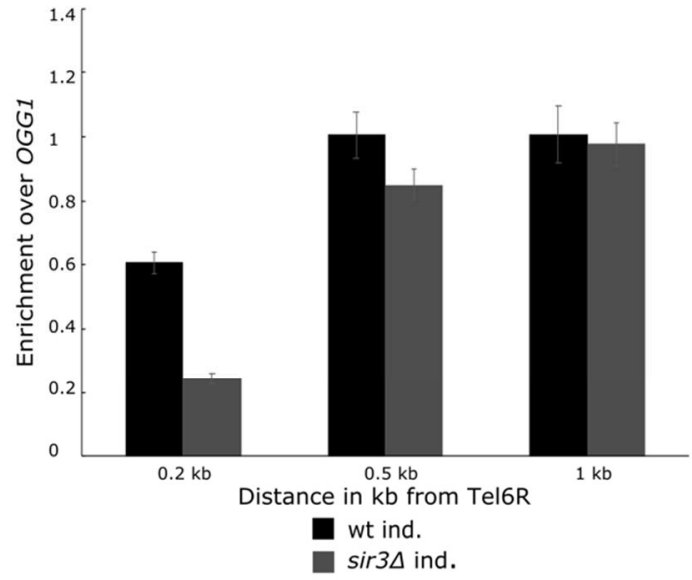

$\mathrm{F}$

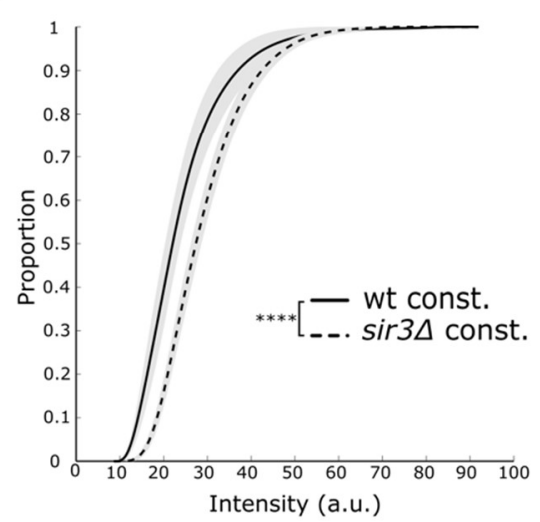

Figure 6. Sir3 spreading counteracted LacI-induced nucleosome eviction. (A) H2A occupancy along the of lys2::lacO $E-A D E 2-I$ locus probed by ChIP-qPCR using an anti-H2A antibody in strains expressing either the GFP-LacIR under the constitutive HIS3 promoter (wt const., yAT2059) or under the GALS promoter (wt ind., yAT2078) in raffinose (no induction), or after $45 \mathrm{~min}, 90 \mathrm{~min}, 180 \mathrm{~min}$, and $1200 \mathrm{~min}$ of galactose induction. (P1), (P2), (P3) (P4) amplicons are respectively located at $3.7 \mathrm{~kb}, 2.2 \mathrm{~kb}, 1 \mathrm{~kb}$, and $0.5 \mathrm{~kb}$ from the left side of the $E$ silencer, and (P5) and (P6) amplicons are located at $3.2 \mathrm{~kb}$ and $6 \mathrm{~kb}$ from the right side of the $E$ silencer, amplicon positions are localized with an asterisk and their respective number on the scheme of the locus The data were normalized over OGG1 (shown as mean \pm s.e.m.; see Table S3 for the number of experiments). (B) $\mathrm{H} 2 \mathrm{~A}$ enrichment over time at the $\mathrm{lacO}(\mathrm{P} 2)$, and nearby the $E$ silencer (P4), in a strain bearing the lys2::lacO E-ADE2-I locus, probed by ChIP-qPCR using an anti-H2A antibody in strains expressing the GFP-LacIR under the inducible 
GALS promoter in the WT (wt ind., yAT2078) and in a sir3 $\Delta$ (sir3 $\Delta$ ind., yAT2370) strain, in raffinose (no induction), or after $45 \mathrm{~min}, 90 \mathrm{~min}, 180 \mathrm{~min}$, and $1200 \mathrm{~min}$ of galactose induction. The data were normalized over OGG1 (shown as mean \pm s.e.m.; see Table S3 for the number of experiments). (C) H2A occupancy at the lacO (P1 and P2) in a strain bearing the lys2::lacO E-ADE2-I, probed by ChIP-qPCR using an anti-H2A antibody, in a strain without LacI expression (wt No LacI, yAT2001) or in strains expressing GFP-LacI under the control of the constitutive HIS3 promoter in the WT (wt const., yAT2000) and in a sir3 $\Delta$ (sir3 $\Delta$ const., yAT2156). The data were normalized over OGG1 (shown as mean \pm s.e.m.; see Table S3 for the number of experiments). (D) $\mathrm{H} 2 \mathrm{~A}$ occupancy at $0.2 \mathrm{~kb}, 0.5 \mathrm{~kb}$, and $1 \mathrm{~kb}$ from the end of telomere $6 \mathrm{R}$ in strains bearing the lys 2::lacO E-ADE2-I locus, probed by ChIP-qPCR using an anti-H2A antibody, and expressing GFP-LacIR under the control of the inducible GALS promoter in the WT (wt ind., yAT2078), and in a sir3 $\triangle$ (sir3 $\triangle$ ind,. yAT2370) strains. The data were normalized over OGG1 (shown as mean \pm s.e.m.; see Table S3 for the number of experiments). (E) Representative fluorescent images (maximal projection of a Z-stack) and their corresponding transmission images of strains bearing the lys2::lacO E-ADE2-I locus and expressing GFP-LacIR under the constitutive HIS3 promoter in the WT (wt const. yAT2059) and the $\operatorname{sir} 3 \Delta$ (sir3 $\Delta$ const., yAT3525) strains. Bar, $2 \mu \mathrm{m}$. (F) Cumulative distributions of the intensity of GFP foci in G1 cells, in strains bearing the lys2::lacO E-ADE2-I locus and expressing GFP-LacIR under the constitutive HIS3 promoter in the WT (wt const. yAT2059) and a sir3 $\Delta$ (sir3 $\Delta$ const., yAT3525) strains. $p>0.01$ is non-significant (ns), $p \leq 0.00001(* * * *)$ and see Table S4 for statistics.

\section{Discussion}

Here we set up an inducible system to monitor silencing establishment at an ectopic locus without affecting SIR protein expression or activity.

In this experimental system, silencing establishment is induced by the binding of the LacI protein on a lacO array located $1.9 \mathrm{~kb}$ away from a reporter of ectopic silencing consisting of the ADE2 gene flanked by the HML silencers inserted at the LYS2 locus [17,18].

Using this system, we observe that LacI binding is accompanied by nucleosome eviction at the lacO array, together with the phosphorylation of $\mathrm{H} 2 \mathrm{~A}$ within the $l a c \mathrm{O}$ array and the neighboring nucleosomes. This is followed by the perinuclear anchoring of the locus and the progressive recruitment of the SIR complex at the $E$ silencer from where it spreads on the flanking regions, including the $l a c O$ array and the $A D E 2$ reporter gene leading to its repression after long-term induction. Another consequence of SIR spreading is the accumulation of H2A-P on the underlying nucleosomes. While the SIR recruitment and the initial phosphorylation of $\mathrm{H} 2 \mathrm{~A}$ are independent events, Sir3 recruitment allows the stabilization of H2A-P. Finally, Sir protein recruitment stabilizes nucleosomes and counteracts LacI binding.

\subsection{LacI Binding Induces H2A Phosphorylation and SIR Recruitment Independently}

The phosphorylation of H2A is the earliest event that we could detect upon LacI binding at the lacO array. This histone modification is triggered by the PI3 kinases Mec1 and Tel1 that are activated upon DNA damage, suggesting that LacI binding induces a DNA or chromatin stress that is sensed by the checkpoint machinery. This chromatin stress could result from the abrupt eviction of nucleosomes upon LacI binding. We observed that this LacI binding induced phosphorylation of H2A depends on both Mec1 and Tel1 with a preponderant impact of Mec1. However, we could not detect the phosphorylation of the downstream effector Rad53 (Figure S5D) indicating a mild activation of the PI3 kinases.

Both Tel1 and the DNA repair factor Mre11 were shown to interact with members of the SIR complex in the two-hybrid system and to promote silencing when artificially tethered to a defective silencer [51]. Hence, Tel1 and Mre11 may trigger silencing through the direct recruitment of the SIR complex. Furthermore, both Mre11- and Tel1-mediated silencing was at least partially dependent on H2A phosphorylation. However, none of these factors is required for silencing establishment in our system (Figure 5). This is consistent with the fact that these factors are also not required for silencing at $H M$ loci, despite being sufficient in Reference [51].

Genome wide, H2A-P is found enriched at SIR bound loci including subtelomeres and $H M$ loci, but also smaller regions located on chromosome arms [5,45,46], and this enrichment decreased in the absence of Sir3. Similarly, we observed a strong decrease in 
H2A-P in the absence of Sir3 after long-term LacI induction. However, the phosphorylation of H2A observed immediately after LacI binding is Sir3 independent. H2A-P and Sir3 recruitment are thus independent events induced by LacI binding at the lacO array. Still, Sir3 spreading stabilizes this otherwise transient mark, leaving a scar of the initial chromatin stress. A similar mechanism could explain the presence of H2A-P at silent loci since natural silencers are made of binding sites for proteins able to destabilize nucleosomes such as Rap1, Abf1, or Orc1. Their binding could cause a chromatin stress, leading to H2A-P that is then stabilized by Sir3 binding. Whether H2A-P and/or Sir3 helps stabilizing these loci will be interesting to study in the future.

\subsection{Silencing Establishment Occurs over Several Cell Cycle}

In contrast to H2A-P, SIR recruitment and silencing establishment is a slow process, with Sir3 and Sir4 binding increasing significantly after $90 \mathrm{~min}$ and accumulating over several cell cycles, leading to gene silencing only after long-term induction $(20 \mathrm{~h})$. This slow establishment of silencing is in contrast with previous reports monitoring silencing establishment at HM loci [24-28,30-32,50]. This difference could be explained by several factors: (i) the chromosomal position of the reporter studied (euchromatic regions $339 \mathrm{~kb}$ away from Tel2R in our system versus subtelomeric HML loci); (ii) the strength of the gene promoter [30]; (iii) the method used to induce silencing establishment. Indeed, in our system silencing is induced while silencing is already established at other loci, whereas previous studies used conditional or inducible alleles of SIR3, thus affecting all silent loci simultaneously. These factors are not mutually exclusive and could even reinforce each other. Because Sir3 is available in a limited amount for silencing establishment, it might be more difficult to recruit Sir3 molecules when they are sequestered at other loci, and the distance to these loci might decrease further the probability to encounter Sir3 molecules [19-22].

\subsection{Anchoring at the Nuclear Periphery Precedes Silencing Establishment}

Silent loci are enriched at the nuclear periphery, whereas the LYS2 locus, when not silent, shows an almost random nuclear localization [17]. Here we observed a progressive accumulation of the locus at the nuclear periphery that precedes silencing establishment. Consistently, this perinuclear anchoring is partially independent of the SIR complex. How LacI binding triggers this change in nuclear localization is not clear. This change in localization that is observed both in G1 and S-G2 phases of the cell cycle, independently of H2A-P, Tel1, or Mec1, requires a high level of LacI expression. This high level of LacI binding results in nucleosome eviction, suggesting that a large nucleosome-free region might be responsible for this relocalization at the nuclear periphery. This SIR-independent perinuclear anchoring could contribute to silencing establishment by bringing the locus in proximity to SIR dense regions, as proposed earlier [21,22].

\subsection{Possible Mechanisms for Silencing Establishment}

What is the mechanism leading to SIR recruitment upon LacI binding? We previously proposed that the replication stress arising from tight DNA-protein interactions could favor heterochromatin formation [17]. Here we show that LacI binding is accompanied by a decrease in histone occupancy along the $\mathrm{lacO}$ array. Binding and unbinding of the LacI protein could increase the turnover of nucleosomes independently of DNA replication, thus leading to the incorporation of unmodified histones that are more permissive to Sir3 binding. In particular, H3K79 tri-methylation, which is widespread in euchromatin, counteracts Sir3 spreading and prevents silencing establishment [5,32,52]. Given the lack of enzyme to erase this histone mark, its removal can be achieved only through turnover of the histones that bear it. Histone turnover occurs mainly during the $S$ phase and was proposed to be a critical cell-cycle-regulated step in silencing establishment [32]. Our data suggest that the dynamics of LacI binding and unbinding could also stimulate histone turnover on the lacO array. 
Nucleosome-depleted regions (NDR) were recently shown to improve silencing establishment and by favoring nucleosome positioning when the distance between two NDRs allows a regular positioning of nucleosomes [53]. However, this effect decreases with the distance between the two NDRs and is, thus, unlikely to be at play in our system where the distance between the $E$ silencer and the $l a c O$ array is $1960 \mathrm{bp}$. Yet, it would be interesting to test whether shortening this distance to a length that could not be evenly divided into nucleosomes would prevent silencing establishment.

As mentioned above, the perinuclear anchoring of the locus that occurs independently of SIR recruitment could also contribute to SIR recruitment by bringing the locus in proximity to the pool of SIR proteins associated with silent loci enriched at the nuclear periphery. In turn, SIR recruitment reinforces the perinuclear anchoring of the locus, feeding a positive loop that increases both SIR recruitment and anchoring at the nuclear periphery.

\subsection{SIR Spreading Stabilizes Nucleosome and Limits the Access to DNA}

The use of our inducible system to study the establishment of silencing at an ectopic locus revealed a positive role for the SIR complex in the stabilization of nucleosomes in vivo. This was also observed at the natural subtelomere $6 \mathrm{R}$ where the sequence immediately adjacent to the telomeric repeats shows a low occupancy in wild-type cells, which is further reduced in the absence of Sir3. A similar effect of heterochromatin in stabilizing nucleosomes has also been reported in S. pombe [54,55]. This stabilization could be a direct consequence of the SIR complex spreading on nucleosomes or an indirect effect owing from the protection of nucleosomes against histone modifiers and remodelers or to a steric hindrance preventing the binding of DNA binding protein. Our observation that SIR spreading at the lacO array reduces LacI binding indicates that SIR bound to nucleosomes occlude the access of DNA binding protein to DNA. This is in good agreement with in vitro studies suggesting that association of the SIR complex to chromatin results in the formation of a fiber characterized by Sir3-dependent stabilization of nucleosomes and occlusion of DNA linkers $[56,57]$.

In conclusion, using our inducible system, we showed that a massive binding of LacI protein leads to nucleosome eviction, inducing a chromatin stress as indicated by the phosphorylation of $\mathrm{H} 2 \mathrm{~A}$ at this locus and its anchoring at the nuclear periphery. This is followed by the recruitment of the SIR complex, which in turn stabilizes nucleosomes, H2A-P, and the perinuclear anchoring of the locus and prevents the access of DNA binding protein to DNA.

Although artificial, this system may reveal a mechanism at play at natural genomic loci. Indeed, natural sites of SIR recruitment such as HM silencers or telomeric repeats are made of combinations of binding sites for protein that are strong nucleosome-displacing factors (NDFs) [6]. Moreover, binding sites for other NDFs such as Ume6, Reb1, or TFIIIC can cooperate with silencer elements to strengthen silencing $[6,17,53]$. Further research will be needed to decipher the precise mechanism by which nucleosome-depleted regions can lead to silencing establishment. 
Supplementary Materials: The following are available online at https: / www.mdpi.com/article/ 10.3390/cells10112810/s1, Figure S1: LacI induced silencing requires Sir3, Sir4 and Sir1, Figure S2: Distribution of intensities and localization of the lys2::lacO E-ADE2-I locus in G1 and S/G2 cells upon LacI induction; Figure S3: Heterochromatin formation takes place over several cell cycles and Sir3 recruitment plateaus after 24h, Figure S4: Perinuclear anchoring is partly Sir3 and Sir4 independent in G1 and S/G2 cells, Figure S5: LacI binding induces a transient H2A phosphorylation without detectable Rad53 phosphorylation, Figure S6: SIR spreading stabilizes nucleosome and limits LacI access to DNA. Table S1: Yeast strains used in this study, Table S2: Primers used in this study, Table S3: Number of experiments for each figure, Table S4: Statistics. References [58,59] are referred to in Supplementary Materials.

Author Contributions: I.L. and I.N. generated strains and performed ChIP experiments. I.L. performed and analyzed the microscopy experiments. M.G. developed the macro for image analyses and conducted the statistical analyses. A.T., I.L. and I.N. contributed to the design of the experiments. A.T., I.L., I.N. and M.G. contributed to the interpretation of the data, the drafting of the figures and the writing of the manuscript. All authors have read and agreed to the published version of the manuscript.

Funding: This research was funded by the ANR (French Agence Nationale de la Recherche) grant numbers ANR-11-LABEX-0044 DEEP, ANR-10-IDEX-0001-02 PSL) and ANR-15-CE12-0007 DNA-Life.

Institutional Review Board Statement: Not applicable.

Informed Consent Statement: Not applicable.

Data Availability Statement: Not applicable.

Acknowledgments: The authors thank the Taddei laboratory for helpful discussions, Myriam Ruault for her critical reading of the manuscript, and Valérie Doye and Benoît Palancade for sharing reagents. The authors also thank the PICT-IBiSA@Pasteur Imaging Facility of the Institut Curie, a member of the France Bioimaging National Infrastructure (ANR-10-INBS-04).

Conflicts of Interest: The authors declare no conflict of interest.

\section{References}

1. Meister, P.; Taddei, A. Building Silent Compartments at the Nuclear Periphery: A Recurrent Theme. Curr. Opin. Genet. Dev. 2013, 23, 96-103. [CrossRef]

2. Bizhanova, A.; Kaufman, P.D. Close to the Edge: Heterochromatin at the Nucleolar and Nuclear Peripheries. Biochim. Biophys. Acta (BBA)-Gene Regul. Mech. 2021, 1864, 194666. [CrossRef] [PubMed]

3. Gartenberg, M.R.; Smith, J.S. The Nuts and Bolts of Transcriptionally Silent Chromatin in Saccharomyces cerevisiae. Genetics 2016, 203, 1563-1599. [CrossRef] [PubMed]

4. Maison, C.; Almouzni, G. HP1 and the Dynamics of Heterochromatin Maintenance. Nat. Rev. Mol. Cell. Biol. $2004,5,296-305$. [CrossRef]

5. Hocher, A.; Ruault, M.; Kaferle, P.; Descrimes, M.; Garnier, M.; Morillon, A.; Taddei, A. Expanding Heterochromatin Reveals Discrete Subtelomeric Domains Delimited by Chromatin Landscape Transitions. Genome Res. 2018, 28, 1867-1881. [CrossRef] [PubMed]

6. Yan, C.; Chen, H.; Bai, L. Systematic Study of Nucleosome-Displacing Factors in Budding Yeast. Mol. Cell 2018, 71, 294-305. [CrossRef]

7. Straight, A.F.; Shou, W.; Dowd, G.J.; Turck, C.W.; Deshaies, R.J.; Johnson, A.D.; Moazed, D. Net1, a Sir2-Associated Nucleolar Protein Required for RDNA Silencing and Nucleolar Integrity. Cell 1999, 97, 245-256. [CrossRef]

8. Radman-Livaja, M.; Ruben, G.; Weiner, A.; Friedman, N.; Kamakaka, R.; Rando, O.J. Dynamics of Sir3 Spreading in Budding Yeast: Secondary Recruitment Sites and Euchromatic Localization: Dynamics of Sir3 Spreadingin Budding Yeast. EMBO J. 2011, 30, 1012-1026. [CrossRef]

9. Ruault, M.; Scolari, V.F.; Lazar-Stefanita, L.; Hocher, A.; Loïodice, I.; Koszul, R.; Taddei, A. Sir3 Mediates Long-Range Chromosome Interactions in Budding Yeast. Genome Res. 2021, 31, 411-425. [CrossRef]

10. Sperling, A.S.; Grunstein, M. Histone H3 N-Terminus Regulates Higher Order Structure of Yeast Heterochromatin. Proc. Natl. Acad. Sci. USA 2009, 106, 13153-13159. [CrossRef]

11. Takahashi, Y.-H.; Schulze, J.M.; Jackson, J.; Hentrich, T.; Seidel, C.; Jaspersen, S.L.; Kobor, M.S.; Shilatifard, A. Dot1 and Histone H3K79 Methylation in Natural Telomeric and HM Silencing. Mol. Cell 2011, 42, 118-126. [CrossRef]

12. Mitsumori, R.; Ohashi, T.; Kugou, K.; Ichino, A.; Taniguchi, K.; Ohta, K.; Uchida, H.; Oki, M. Analysis of Novel Sir3 Binding Regions in Saccharomyces cerevisiae. J. Biochem. 2016, 160, 11-17. [CrossRef] 
13. Buck, S.W.; Shore, D. Action of a RAP1 Carboxy-Terminal Silencing Domain Reveals an Underlying Competition between HMR and Telomeres in Yeast. Genes Dev. 1995, 9, 370-384. [CrossRef]

14. Smith, J.S.; Brachmann, C.B.; Pillus, L.; Boeke, J.D. Distribution of a Limited Sir2 Protein Pool Regulates the Strength of Yeast RDNA Silencing and Is Modulated by Sir4p. Genetics 1998, 149, 1205-1219. [CrossRef]

15. Michel, A.H. Spontaneous RDNA Copy Number Variation Modulates Sir2 Levels and Epigenetic Gene Silencing. Genes Dev. 2005, 19, 1199-1210. [CrossRef] [PubMed]

16. Taddei, A.; Gasser, S.M. Structure and Function in the Budding Yeast Nucleus. Genetics 2012, 192, 107-129. [CrossRef] [PubMed]

17. Dubarry, M.; Loiodice, I.; Chen, C.L.; Thermes, C.; Taddei, A. Tight Protein-DNA Interactions Favor Gene Silencing. Genes Dev. 2011, 25, 1365-1370. [CrossRef] [PubMed]

18. Maillet, L.; Gaden, F.; Brevet, V.; Fourel, G.; Martin, S.G.; Dubrana, K.; Gasser, S.M.; Gilson, E. Ku-deficient Yeast Strains Exhibit Alternative States of Silencing Competence. EMBO Rep. 2001, 2, 203-210. [CrossRef]

19. Marcand, S.; Buck, S.W.; Moretti, P.; Gilson, E.; Shore, D. Silencing of Genes at Nontelomeric Sites in Yeast Is Controlled by Sequestration of Silencing Factors at Telomeres by Rap 1 Protein. Genes Dev. 1996, 10, 1297-1309. [CrossRef]

20. Maillet, L.; Boscheron, C.; Gotta, M.; Marcand, S.; Gilson, E.; Gasser, S.M. Evidence for Silencing Compartments within the Yeast Nucleus: A Role for Telomere Proximity and Sir Protein Concentration in Silencer-Mediated Repression. Genes Dev. 1996, 10, 1796-1811. [CrossRef]

21. Taddei, A.; Van Houwe, G.; Nagai, S.; Erb, I.; van Nimwegen, E.; Gasser, S.M. The Functional Importance of Telomere Clustering: Global Changes in Gene Expression Result from SIR Factor Dispersion. Genome Res. 2009, 19, 611-625. [CrossRef]

22. Andrulis, E.D.; Neiman, A.M.; Zappulla, D.C.; Sternglanz, R. Perinuclear Localization of Chromatin Facilitates Transcriptional Silencing. Nature 1998, 394, 592-595. [CrossRef] [PubMed]

23. Katan-Khaykovich, Y.; Struhl, K. Heterochromatin Formation Involves Changes in Histone Modifications over Multiple Cell Generations. EMBO J. 2005, 24, 2138-2149. [CrossRef] [PubMed]

24. Osborne, E.A.; Dudoit, S.; Rine, J. The Establishment of Gene Silencing at Single-Cell Resolution. Nat. Genet. 2009, 41, 800-806. [CrossRef] [PubMed]

25. Osborne, E.A.; Hiraoka, Y.; Rine, J. Symmetry, Asymmetry, and Kinetics of Silencing Establishment in Saccharomyces cerevisiae Revealed by Single-Cell Optical Assays. Proc. Natl. Acad. Sci. USA 2011, 108, 1209-1216. [CrossRef]

26. Miller, A.M.; Nasmyth, K.A. Role of DNA Replication in the Repression of Silent Mating Type Loci in Yeast. Nature 1984, 312, 247-251. [CrossRef]

27. Lau, A. Cell-Cycle Control of the Establishment of Mating-Type Silencing in S. cerevisiae. Genes Dev. 2002, 16, 2935-2945. [CrossRef]

28. Martins-Taylor, K.; Sharma, U.; Rozario, T.; Holmes, S.G. H2A.Z (Htz1) Controls the Cell-Cycle-Dependent Establishment of Transcriptional Silencing at Saccharomyces cerevisiae Telomeres. Genetics 2011, 187, 89-104. [CrossRef]

29. Martins-Taylor, K.; Dula, M.L.; Holmes, S.G. Heterochromatin Spreading at Yeast Telomeres Occurs in M Phase. Genetics 2004, 168, 65-75. [CrossRef]

30. Ren, J.; Wang, C.-L.; Sternglanz, R. Promoter Strength Influences the S Phase Requirement for Establishment of Silencing at the Saccharomyces cerevisiae Silent Mating Type Loci. Genetics 2010, 186, 551-560. [CrossRef]

31. Lazarus, A.G.; Holmes, S.G. A Cis -Acting TRNA Gene Imposes the Cell Cycle Progression Requirement for Establishing Silencing at the HMR Locus in Yeast. Genetics 2011, 187, 425-439. [CrossRef]

32. Goodnight, D.; Rine, J. S-Phase-Independent Silencing Establishment in Saccharomyces cerevisiae. eLife 2020, 9, e58910. [CrossRef]

33. Thomas, B.J.; Rothstein, R. Elevated Recombination Rates in Transcriptionally Active DNA. Cell 1989, 56, 619-630. [CrossRef]

34. Winston, F.; Dollard, C.; Ricupero-Hovasse, S.L. Construction of a Set of Convenient Saccharomyces cerevisiae Strains That Are Isogenic to S288C. Yeast 1995, 11, 53-55. [CrossRef] [PubMed]

35. Longtine, M.S.; Iii, A.M.; Demarini, D.J.; Shah, N.G.; Wach, A.; Brachat, A.; Philippsen, P.; Pringle, J.R. Additional Modules for Versatile and Economical PCR-Based Gene Deletion and Modification in Saccharomyces cerevisiae. Yeast 1998, 14, 953-961. [CrossRef]

36. Janke, C.; Magiera, M.M.; Rathfelder, N.; Taxis, C.; Reber, S.; Maekawa, H.; Moreno-Borchart, A.; Doenges, G.; Schwob, E.; Schiebel, E.; et al. A Versatile Toolbox for PCR-Based Tagging of Yeast Genes: New Fluorescent Proteins, More Markers and Promoter Substitution Cassettes. Yeast 2004, 21, 947-962. [CrossRef] [PubMed]

37. Nistér, D.; Stewénius, H. Linear Time Maximally Stable Extremal Regions. In Proceedings of the Computer Vision-ECCV 2008, Marseille, France, 12-18, October 2008; Forsyth, D., Torr, P., Zisserman, A., Eds.; Lecture Notes in Computer Science; Springer: Berlin/Heidelberg, Germany, 2008; Volume 5303, pp. 183-196, ISBN 978-3-540-88685-3.

38. Kruskal, W.H. Use of Ranks in One-Criterion Variance Analysis. J. Am. Stat. Assoc. 1952, 40. [CrossRef]

39. Tukey, J.W. Comparing Individual Means in the Analysis of Variance. Biometrics 1949, 5, 99. [CrossRef] [PubMed]

40. Kitamura, E.; Blow, J.J.; Tanaka, T.U. Live-Cell Imaging Reveals Replication of Individual Replicons in Eukaryotic Replication Factories. Cell 2006, 125, 1297-1308. [CrossRef]

41. Wang, Y.; Liu, C.L.; Storey, J.D.; Tibshirani, R.J.; Herschlag, D.; Brown, P.O. Precision and Functional Specificity in MRNA Decay. Proc. Natl. Acad. Sci. USA 2002, 99, 5860-5865. [CrossRef] 
42. Grigull, J.; Mnaimneh, S.; Pootoolal, J.; Robinson, M.D.; Hughes, T.R. Genome-Wide Analysis of MRNA Stability Using Transcription Inhibitors and Microarrays Reveals Posttranscriptional Control of Ribosome Biogenesis Factors. Mol. Cell. Biol. 2004, 24, 5534-5547. [CrossRef]

43. Miller, C.; Schwalb, B.; Maier, K.; Schulz, D.; Dümcke, S.; Zacher, B.; Mayer, A.; Sydow, J.; Marcinowski, L.; Dölken, L.; et al. Dynamic Transcriptome Analysis Measures Rates of MRNA Synthesis and Decay in Yeast. Mol. Syst. Biol. 2011, 7, 458. [CrossRef] [PubMed]

44. Geisberg, J.V.; Moqtaderi, Z.; Fan, X.; Ozsolak, F.; Struhl, K. Global Analysis of MRNA Isoform Half-Lives Reveals Stabilizing and Destabilizing Elements in Yeast. Cell 2014, 156, 812-824. [CrossRef]

45. Kitada, T.; Schleker, T.; Sperling, A.S.; Xie, W.; Gasser, S.M.; Grunstein, M. ГH2A Is a Component of Yeast Heterochromatin Required for Telomere Elongation. Cell. Cycle 2011, 10, 293-300. [CrossRef]

46. Szilard, R.K.; Jacques, P.-É.; Laramée, L.; Cheng, B.; Galicia, S.; Bataille, A.R.; Yeung, M.; Mendez, M.; Bergeron, M.; Robert, F.; et al. Systematic Identification of Fragile Sites via Genome-Wide Location Analysis of $\gamma$-H2AX. Nat. Struct. Mol. Biol. 2010, 17, 299-305. [CrossRef]

47. Colombo, C.V.; Gnugnoli, M.; Gobbini, E.; Longhese, M.P. How Do Cells Sense DNA Lesions? Biochem. Soc. Trans. 2020, 48, 677-691. [CrossRef] [PubMed]

48. Ravindra, A.; Weiss, K.; Simpson, R.T. High-Resolution Structural Analysis of Chromatin at Specific Loci: Saccharomyces cerevisiae Silent Mating-Type Locus HMRa. Mol. Cell. Biol. 1999, 19, 7944-7950. [CrossRef]

49. Weiss, K.; Simpson, R.T. High-Resolution Structural Analysis of Chromatin at Specific Loci: Saccharomyces cerevisiae Silent Mating Type Locus HML $\alpha$. Mol. Cell. Biol. 1998, 18, 5392-5403. [CrossRef]

50. Zou, Y.; Yu, Q.; Bi, X. Asymmetric Positioning of Nucleosomes and Directional Establishment of Transcriptionally Silent Chromatin by Saccharomyces cerevisiae Silencers. Mol. Cell. Biol. 2006, 26, 7806-7819. [CrossRef] [PubMed]

51. Kirkland, J.G.; Peterson, M.R.; Still, C.D.; Brueggeman, L.; Dhillon, N.; Kamakaka, R.T. Heterochromatin Formation via Recruitment of DNA Repair Proteins. MBoC 2015, 26, 1395-1410. [CrossRef] [PubMed]

52. Kitada, T.; Kuryan, B.G.; Tran, N.N.H.; Song, C.; Xue, Y.; Carey, M.; Grunstein, M. Mechanism for Epigenetic Variegation of Gene Expression at Yeast Telomeric Heterochromatin. Genes Dev. 2012, 26, 2443-2455. [CrossRef]

53. Saxton, D.S.; Rine, J. Nucleosome Positioning Regulates the Establishment, Stability, and Inheritance of Heterochromatin in Saccharomyces cerevisiae. Proc. Natl. Acad. Sci. USA 2020, 117, 27493-27501. [CrossRef]

54. Garcia, J.F.; Dumesic, P.A.; Hartley, P.D.; El-Samad, H.; Madhani, H.D. Combinatorial, Site-Specific Requirement for Heterochromatic Silencing Factors in the Elimination of Nucleosome-Free Regions. Genes Dev. 2010, 24, 1758-1771. [CrossRef]

55. Emden, T.S.; Forn, M.; Forné, I.; Sarkadi, Z.; Capella, M.; Martín Caballero, L.; Fischer-Burkart, S.; Brönner, C.; Simonetta, M.; Toczyski, D.; et al. Shelterin and Subtelomeric DNA Sequences Control Nucleosome Maintenance and Genome Stability. EMBO Rep. 2019, 20. [CrossRef] [PubMed]

56. Swygert, S.G.; Senapati, S.; Bolukbasi, M.F.; Wolfe, S.A.; Lindsay, S.; Peterson, C.L. SIR Proteins Create Compact Heterochromatin Fibers. Proc. Natl. Acad. Sci. USA 2018, 115, 12447-12452. [CrossRef] [PubMed]

57. Kueng, S.; Oppikofer, M.; Gasser, S.M. SIR Proteins and the Assembly of Silent Chromatin in Budding Yeast. Annu. Rev. Genet. 2013, 47, 275-306. [CrossRef] [PubMed]

58. Ruault, M.; De Meyer, A.; Loïodice, I.; Taddei, A. Clustering heterochromatin: Sir3 promotes telomere clustering independently of silencing in yeast. J. Cell Biol. 2011, 192, 417-431. [CrossRef]

59. Masumoto, H.; Hawke, D.; Kobayashi, R.; Verreault, A. A role for cell-cycle-regulated histone H3 lysine 56 acetylation in the DNA damage response. Nature 2005, 436, 294-298. [CrossRef] 